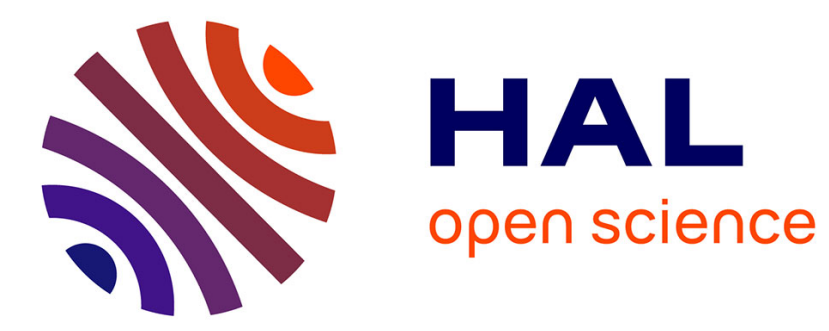

\title{
Discrete asymptotic equations for long wave propagation
}

S Bellec, Mathieu Colin, Mario Ricchiuto

\section{To cite this version:}

S Bellec, Mathieu Colin, Mario Ricchiuto. Discrete asymptotic equations for long wave propagation.

[Research Report] RR-8806, Inria Bordeaux Sud-Ouest. 2015, pp.29. hal-01224157

\section{HAL Id: hal-01224157 \\ https://hal.inria.fr/hal-01224157}

Submitted on 4 Nov 2015

HAL is a multi-disciplinary open access archive for the deposit and dissemination of scientific research documents, whether they are published or not. The documents may come from teaching and research institutions in France or abroad, or from public or private research centers.
L'archive ouverte pluridisciplinaire HAL, est destinée au dépôt et à la diffusion de documents scientifiques de niveau recherche, publiés ou non, émanant des établissements d'enseignement et de recherche français ou étrangers, des laboratoires publics ou privés. 


\section{Discrete asymptotic} equations

for long wave propagation

S. Bellec, M. Colin , M. Ricchiuto

\section{RESEARCH}

\section{REPORT}

$\mathrm{N}^{\circ} 8806$

November 2015

Project-Teams CARDAMOM 



\title{
Cnzía
}

\section{Discrete asymptotic equations for long wave propagation}

\author{
S. Bellec *, M. Colin *, M. Ricchiuto * \\ Project-Teams CARDAMOM \\ Research Report $\mathrm{n}^{\circ} 8806$ - November $2015-29$ pages
}

\begin{abstract}
In this paper, we present a new systematic method to obtain some discrete numerical models for incompressible free-surface flows. The method consists in first discretizing the Euler equations with respect to one variable, keeping the other ones unchanged and then performing an asymptotic analysis on the resulting system. For the sake of simplicity, we choose to illustrate this method in the context of the Peregrine asymptotic regime, that is we propose an alternative numerical scheme for the so-called Peregrine equations. We then study the linear dispersion characteristics of our new scheme and present several numerical experiments to measure the relevance of the method.
\end{abstract}

Key-words: Asymptotic equations, discrete asymptotics, Boussinesq models, finite elements

* Inria BSO, Team CARDAMOM

\section{RESEARCH CENTRE \\ BORDEAUX - SUD-OUEST}

351, Cours de la Libération

Bâtiment A 29

33405 Talence Cedex 


\section{Discrete asymptotic equations for long wave propagation}

Résumé : Dans cet article on présente une nouvelle derivation systématique de modèles numériques pour écoulements à surface libre. La méthode utilisé consiste en discretiser les équations d'Euler dans la direction horizontale et apres en introduisant une expansion asymptotique des equations discrètes obtenues. Pour simplicité dans ce travail on considère que le cas d'un système analogue aus équations de Peregrine. On analyse les propriétés de dispersion et du nouveau modele discret en montrant une nette amélioration par rapport à la discretisation directe du système asymptotique continu.

Mots-clés : Asymptotic equations, discrete asymptotics, Boussinesq models, finite elements 


\section{Contents}

1 Introduction 3

2 Setting, notations and main result. 4

3 Galerkin discretization of the Peregrine equations 6

4 A new setting for deriving discrete asymptotic models. $\quad 8$

4.1 Semi-discretization of the 2D-Euler equations in non-dimensional form. . . 8

4.2 Asymptotic expansions on the velocity $U$ and the pressure $p . \ldots \ldots$

4.3 Depth-averaged equations . . . . . . . . . . . . . . . . . 13

5 Study of the linear dispersion characteristics. $\quad 16$

5.1 Linear characteristics of the new numerical model. . . . . . . . . . . . . 16

5.2 Linear characteristics of the classical Peregrine model. . . . . . . . . . . 20

5.3 Analysis of the computations. . . . . . . . . . . . . . . . . 21

5.3 .1 Phase velocity . . . . . . . . . . . . . . . . . . 21

5.3 .2 Linear shoaling test . . . . . . . . . . . . . . . 23

6 Numerical experiments 24

6.1 Soliton propagation . . . . . . . . . . . . . . . . . . 25

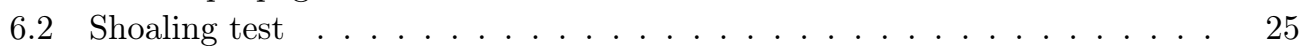

$\begin{array}{lll}7 & \text { Conclusions and perspectives } & 27\end{array}$

\section{Introduction}

Wave transformation in near shore zone is well-described by the incompressible Euler equations. Due to their three-dimensional character, these equations are often too costly if one wants to perform numerical experiments, and often replaced by asymptotic depthaveraged models known as Boussinesq equations. A major characteristic of these models is their ability to describe the dispersive behaviour of wave propagation. Generally, the linear and nonlinear dispersion characteristics of the waves represented by Boussinesq models can be improved by including high order contributions in the double asymptotic expansion in terms of the ratios wave height over wave length (dispersion) and wave height over depth (nonlinearity) [12]. Other techniques to improve the linear dispersion characteristics involve the inclusion of extra dispersive differential terms, derived either from a linear wave equation $[2,13]$, or by replacing depth-averaged values by point values at a properly chosen depth [14]. When numerically simulating the propagation of long waves, the physics represented by these continuous systems of Partial Differential Equations (PDE) is further filtered by the numerical scheme, and in particular by the form of the truncation error. For most of Boussinesq models, the task of designing an accurate numerical discretization is a nontrivial one, due to the presence of dispersion terms. Several approaches exist in literature, each with its own advantages and drawbacks. For details, the interested reader may refer to [4, 8, 11, 16], to the review [5], and references there in. The objective of this paper is to study the interaction scheme-PDE and to propose a framework to obtain new schemes with improved characteristics w.r.t. existing approaches. For this purpose, we introduce a new scheme reversing the model derivation procedure. More precisely, we propose to discretize partially the incompressible Euler equations with respect to one direction using a finite element method, and then follow Peregrine's derivation procedure. This new paradigm leads to a very promising scheme 
with nice dispersion properties.

The paper is organized as follows. In Section 2 we introduce some notation and recall our main result. The finite element discretization of a well known Boussinesq system, and most of the algebraic operators involved in our analysis are presented in Section 3. In Section 4 , we detail the derivation of the new numerical scheme. The theoretical analysis of these discrete asymptotic models is presented in Section 5. Finally, Section 6 presents a numerical evaluation of the performances of the schemes, confirming our theoretical results. The paper is ended by an overlook of future developments related to the new approach proposed.

\section{Setting, notations and main result.}

Before going further, let us introduce some notations. For simplicity, in this article, we only deal with 2 -D and $1-\mathrm{D}$ problems. Denote by $(x, z)$ respectively the horizontal and the vertical spatial dimension. Denote by $d(x)$ the depth at still water and $\eta(t, x)$ the surface elevation from its rest position. The total depth is then $h(t, x)=d(x)+\eta(t, x)$ (see Figure 1).

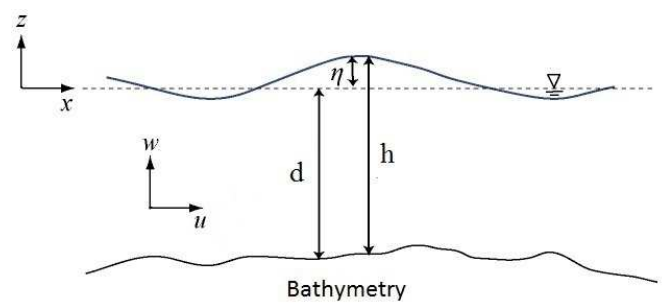

Figure 1: Sketch of the free surface flow problem, main parameters description.

Let $a$ be a typical wave amplitude, $d_{0}$ a reference water depth and $\lambda$ a typical wavelength. In view of performing an asymptotic analysis, we introduce the nonlinearity parameter $\varepsilon$ and the dispersion parameter $\sigma$ defined by

$$
\varepsilon=\frac{a}{d_{0}}, \sigma=\frac{d_{0}}{\lambda}
$$

Under the Boussinesq hypothesis $\varepsilon=\mathcal{O}\left(\sigma^{2}\right)$, Peregrine (see [15]) first derived, from the Euler equations, the following standard system of Boussinesq's type

$$
\begin{aligned}
& \eta_{t}+[h \bar{u}]_{x}=0, \\
& \bar{u}_{t}+\bar{u} \bar{u}_{x}+g \eta_{x}+\left(\frac{d^{2}}{6} \bar{u}_{t x x}-\frac{d}{2}[d \bar{u}]_{t x x}\right)=0 .
\end{aligned}
$$

The model describes the evolution of the depth-averaged velocity $\bar{u}$ and the surface elevation $\eta$ within an accuracy of $\mathcal{O}\left(\varepsilon \sigma^{2}, \sigma^{4}\right)$ w.r.t. the Euler equations. The set of Equations (1) is now well-understood from the computation point of view and a classical numerical scheme can be obtained by using the finite element method in the following stetting. On an intervall $[r, s]$, we introduce a set of nodes

$$
r=x_{0}<x_{1}<\cdots<x_{N}=s,
$$


where, for simplicity, we take a constant space step $\Delta_{x}=x_{j+1}-x_{j}, \forall j \in\{0, \ldots, N\}$. We denote by $E, \bar{U}, D$ and $H$ the vectors of the nodal values of $\eta, \bar{u}, d$ and $h$. Similarly to what has been done in $[18,17]$ (cf. also [16] and references therein), we apply the $\mathbb{P}_{1}$ Galerkin method to approximate the variational form of (1). In particular, we denote by $\left\{\varphi_{j}\right\}_{0 \leq j \leq N}$ the standard piecewise linear continuous Lagrange basis, and introduce the discrete velocity, wave height and depth polynomials as follows

$$
\bar{u}_{\Delta}(t, x)=\sum_{j=0}^{N} \bar{u}_{j}(t) \varphi_{j}(x), \eta_{\Delta}(t, x)=\sum_{j=0}^{N} \eta_{j}(t) \varphi_{j}(x), d_{\Delta}(x)=\sum_{j=0}^{N} d_{j} \varphi_{j}(x) .
$$

The Galerkin approximation of (1), under the hypothesis of exact integration w.r.t. all the discrete polynomials involved, can be written in a compact matrix form

$$
\begin{aligned}
& \mathcal{M} E_{t}+\frac{1}{3}(2 \mathcal{N}(H \diamond \bar{U})+H \diamond(\mathcal{N} \bar{U})+\bar{U} \diamond(\mathcal{N} H))=0, \\
& \mathcal{M} \bar{U}_{t}+\frac{1}{3}\left(\mathcal{N}\left(\bar{U}^{2}\right)+\bar{U} \diamond(\mathcal{N} \bar{U})\right)+g \mathcal{N} E-\frac{1}{6}\left\{D ; \bar{U}_{t}\right\}=0,
\end{aligned}
$$

where the operation $A \diamond B$ represents, for two given vectors $A=\left(a_{i}\right)_{0 \leq i \leq N}$ and $B=$ $\left(b_{i}\right)_{0 \leq i \leq N}$, the column vector $\left(a_{i} b_{i}\right)_{0 \leq i \leq N}$ (note that, given a column vector $A$, notation $A^{2}$ simplifies $\left.A \diamond A\right)$. Remark that the matrices $\mathcal{M}, \mathcal{N}$ and $\mathcal{Q}$ are the usual mass, derivation, and stiffness matrices arising in the Galerkin discretization and are detailed in the next section. Moreover, for given columns vectors $A$ and $B$, we set

$$
\{A ; B\}=\mathcal{Q}\left(A^{2} \diamond B\right)+D \diamond(\mathcal{Q}(A \diamond B)+2(A \diamond B) \diamond(\mathcal{Q} A)-B \diamond(\mathcal{Q} A) .
$$

The aim of this paper is to propose a systematic method to obtain new numerical models describing free surface flows. It is based on the following idea : reverse the model derivation procedure and first discretize partially the Incompressible Euler equations and then derive fully discrete asymptotic equations by performing an asymptotic analysis. To illustrate the potential of this idea, we apply this method to the couple Euler-Peregrine equations by applying the Galerkin method to the variable $x$ and then performing the asymptotic analysis of Peregrine's type to the resulting equations. Of course, when one deals with non-linear equations, this procedure does not commute with the classical one. In this paper, for simplicity, we deal with periodic boundary conditions. Note that our strategy can be adapted directly to the Dirichlet boundary conditions, since the crucial property (50) hold for this two kind of conditions. This strategy is similar to the one proposed for compressible multiphase flows in [1]. As shown in the detailed derivation of the next sections, the new procedure leads to the following discrete equations approximating the discretized Euler system within an accuracy of $\mathcal{O}\left(\varepsilon \sigma^{2}, \sigma^{4}\right)$

$$
\begin{aligned}
& \mathcal{M} E_{t}+\mathcal{M}[H ; \bar{U}]=0 \\
& \mathcal{M} \bar{U}_{t}+\frac{1}{3}\left(\mathcal{N}\left(\bar{U}^{2}\right)+\bar{U} \diamond(\mathcal{N} \bar{U})\right)+g \mathcal{N} E+\mathcal{M}\left(\frac{D^{2}}{6} \diamond\left(\mathcal{K}^{2} \bar{U}_{t}\right)-\frac{D}{2} \diamond\left(\mathcal{K}\left[D ; \bar{U}_{t}\right]\right)\right)=0
\end{aligned}
$$

having introduced the operator $[\cdot ; \cdot]$ defined by

$$
[A ; B]=A \diamond(\mathcal{K} B)+\frac{1}{3}\left[\mathcal{K}(A \diamond B)-\mathcal{M}^{-1}(A \diamond(\mathcal{N} B))+2 \mathcal{M}^{-1}(B \diamond(\mathcal{N} A))\right],
$$

with $\mathcal{K}=\mathcal{M}^{-1} \mathcal{N}$. We see that, while involving similar algebraic operations, the new discretization is different from the classical ones, even for a simple case like Peregrine equations. The main differences are found in the treatment of the third order derivatives terms as well as in the nonlinear ones in the continuity (wave height) equation. We 
will show that scheme (3) also converges to an approximation of the Perigrine equations. However, both the linear phase relation, and the lienar shoaling gradient provided by (3) (see Section 5 and 6) are substantially closer to the exact ones than those given by (4). this gain in accuracy is confirmed by the numerical tests showing a reduction of the error of 5 to 10 times w.r.t. scheme (4). In the sequel, we show how to derive the scheme (3) and prove that not only they are consistent with system (1), but that they represent a substantial improvement w.r.t. the scheme obtained by discretizing directly the asymptotic equations (1).

The paper is organized as follows. In Section 3, we recall the classic discretization of Peregrine system (1). In Section 4, we detail the derivation of the new numerical scheme. In Section 5, we present the study of the exact linear dispersion characteristics for the two schemes presented before. Then, we compare the results with the theoretical characteristics of Peregrine model. To conclude, in Section 6, we present two numerical computations in order to evaluate the accuracy of the new scheme.

\section{Galerkin discretization of the Peregrine equations}

We start by deriving a spatially discretized version of (1) using the Galerkin finite element scheme. Since we deal with varying bathymetry, we first rewrite (1) by developing the derivatives terms

$$
\begin{gathered}
\eta_{t}+[(d+\eta) \bar{u}]_{x}=0 \\
\bar{u}_{t}+\bar{u} \bar{u}_{x}+g \eta_{x}-\frac{d^{2}}{3} \bar{u}_{t x x}-d d_{x} \bar{u}_{t x}-\frac{d d_{x x}}{2} \bar{u}_{t}=0 .
\end{gathered}
$$

According to the Galerkin method (see [7]), we multiply (5) by the Galerkin basis $\phi_{i}$ and we integrate the resulting equation between $x_{i-1}$ and $x_{i+1}$, for $i \in\{0, \ldots, N\}$, with the convention $x_{-1}=x_{N}$ and $x_{N+1}=x_{0}$ coming from the choice of the periodic boundary conditions to obtain

$$
\int_{x_{i-1}}^{x_{i+1}} \eta_{\Delta}(t, x) \varphi_{i}(x) d x-\int_{x_{i-1}}^{x_{i+1}}\left(d_{\Delta}(x)+\eta_{\Delta}(t, x)\right) \bar{u}_{\Delta}(t, x) \varphi_{i}^{\prime}(x) d x=0 .
$$

In the $\mathbb{P}_{1}$ case, evaluating all the integrals exactly w.r.t. the linear spatial variation of $d_{\Delta}, \eta_{\Delta}$, and $\bar{u}_{\Delta}$, we derive from (7), using the decomposition (2) and evaluating all the integrals involving $\phi_{i}$, for all $i \in\{0, \ldots, N\}$

$$
\begin{aligned}
& \frac{\Delta_{x}}{6} \frac{\partial}{\partial t}\left(\eta_{i+1}+4 \eta_{i}+\eta_{i-1}\right) \\
& +\frac{1}{3}\left(2 \frac{h_{i+1} \bar{u}_{i+1}-h_{i-1} \bar{u}_{i-1}}{2}+h_{i} \frac{\bar{u}_{i+1}-\bar{u}_{i-1}}{2}+\bar{u}_{i} \frac{h_{i+1}-h_{i-1}}{2}\right)=0 .
\end{aligned}
$$

As already mentioned, for simplicity, the boundary conditions are assumed to be periodic, although other conditions can be embedded in the analysis. Applying the same procedure to Equation (6), one has, performing integration by parts, for all $i \in\{0, \ldots, N\}$,

$$
\begin{aligned}
& \int_{x_{i-1}}^{x_{i+1}} \frac{d}{d t} \bar{u}_{\Delta}(t, x) \varphi_{i}(x) d x-\int_{x_{i-1}}^{x_{i+1}}\left(\frac{\bar{u}_{\Delta}^{2}(t, x)}{2}+g \eta_{\Delta}(t, x)\right) \varphi_{i}^{\prime}(x) d x \\
+ & \int_{x_{i-1}}^{x_{i+1}}\left\{-\varphi_{i}(x) d d_{\Delta}^{\prime}\left(\bar{u}_{\Delta}\right)_{t x}+\frac{\left(d_{\Delta}^{2}(x) \varphi_{i}(x)\right)_{x}}{3}\left(\bar{u}_{\Delta}(t, x)\right)_{t x}+\frac{\left(\varphi_{i}(x) d_{\Delta}(x)\left(\bar{u}_{\Delta}(t, x)\right)_{t}\right)_{x}}{2} d_{\Delta}^{\prime}\right\} d x \\
& =0 .
\end{aligned}
$$


Plugging relations (2) in (9), one derives after the computation of the different integrals, for all $i \in\{0, \ldots, N\}$

$$
\begin{gathered}
\frac{\Delta_{x}}{6} \frac{d}{d t}\left(\bar{u}_{i+1}+4 \bar{u}_{i}+\bar{u}_{i-1}\right)+\frac{1}{3}\left[\frac{\bar{u}_{i+1}^{2}-\bar{u}_{i-1}^{2}}{2}+\bar{u}_{i} \frac{\bar{u}_{i+1}-\bar{u}_{i-1}}{2}\right]+g \frac{\eta_{i+1}-\eta_{i-1}}{2} \\
-\frac{1}{6 \Delta_{x}} \frac{d}{d t}\left[2 d_{i} \bar{u}_{i}\left(d_{i+1}-2 d_{i}+d_{i-1}\right)+\left(d_{i+1}^{2} \bar{u}_{i+1}-2 d_{i}^{2} \bar{u}_{i}+d_{i-1}^{2} \bar{u}_{i-1}\right)\right. \\
\left.+d_{i}\left(d_{i+1} \bar{u}_{i+1}-2 d_{i} \bar{u}_{i}+d_{i-1} \bar{u}_{i-1}\right)-\bar{u}_{i}\left(d_{i+1}^{2}-2 d_{i}^{2}+d_{i-1}^{2}\right)\right]=0 .
\end{gathered}
$$

In order to rewrite the discrete equations (9) in a simpler and more compact form, we define the mass matrix $\mathcal{M}$, the first order derivative matrix $\mathcal{N}$ and the second order derivative matrix $\mathcal{Q}$ (which represents the laplacian operator with periodic boundary conditions) as follows

$$
\begin{gathered}
\mathcal{M}=\frac{1}{6}\left(\begin{array}{cccccc}
4 & 1 & 0 & \cdots & 0 & 1 \\
1 & \ddots & \ddots & \ddots & & 0 \\
0 & \ddots & \ddots & \ddots & \ddots & \vdots \\
\vdots & \ddots & \ddots & \ddots & \ddots & 0 \\
0 & & \ddots & \ddots & \ddots & 1 \\
1 & 0 & \cdots & 0 & 1 & 4
\end{array}\right), \mathcal{N}=\frac{1}{2 \Delta_{x}}\left(\begin{array}{ccccccc}
0 & 1 & 0 & \cdots & 0 & -1 \\
-1 & \ddots & \ddots & \ddots & & 0 \\
0 & \ddots & \ddots & \ddots & \ddots & \vdots \\
\vdots & \ddots & \ddots & \ddots & \ddots & 0 \\
0 & & \ddots & \ddots & \ddots & 1 \\
1 & 0 & \cdots & 0 & -1 & 0
\end{array}\right) \\
\mathcal{Q}=\frac{1}{\Delta_{x}^{2}}\left(\begin{array}{cccccc}
-2 & 1 & 0 & \cdots & 0 & 1 \\
1 & \ddots & \ddots & \ddots & & 0 \\
0 & \ddots & \ddots & \ddots & \ddots & \vdots \\
\vdots & \ddots & \ddots & \ddots & \ddots & 0 \\
0 & & \ddots & \ddots & \ddots & 1 \\
1 & 0 & \cdots & 0 & 1 & -2
\end{array}\right)
\end{gathered}
$$

In addition, we introduce the following column vectors

$$
\bar{U}=\left(\bar{u}_{i}\right)_{0 \leq i \leq N}, E=\left(\eta_{i}\right)_{0 \leq i \leq N}, D=\left(d_{i}\right)_{0 \leq i \leq N}, H=D+E .
$$

Equation (8) can thus be written as

$$
\Delta_{x} \frac{d}{d t} \mathcal{M} E+\frac{1}{3}\left[2 \mathcal{N}\left(h_{i} \bar{u}_{i}\right)_{i \in\{0, . ., N\}}+\left(h_{i}(\mathcal{N} \bar{U})_{i}\right)_{i \in\{0, . ., N\}}+\left(\bar{u}_{i}(\mathcal{N} H)_{i}\right)_{i \in\{0, . ., N\}}\right]=0 .
$$

To further simplify the notation for given columns vectors $A=\left(a_{i}\right)_{0 \leq i \leq N}$ and $B=$ $\left(b_{i}\right)_{0 \leq i \leq N}$, we introduce the operator $\diamond$ :

$$
\begin{aligned}
\mathbb{R}^{N} \times \mathbb{R}^{N} & \rightarrow \mathbb{R}^{N} \\
(A, B) & \rightarrow A \diamond B:=\left(a_{i} b_{i}\right)_{0 \leq i \leq N}
\end{aligned}
$$

In the sequel, for simplicity $A^{2}$ simplifies $A \diamond A$. As an example, the vector $\left(h_{i}(\mathcal{N} \bar{U})_{i}\right)_{i \in\{1, . ., n\}}$ can be rewritten as $H \diamond(\mathcal{N} \bar{U})$. Owning this notation, the discrete equations (8)-(10) can be compactly written as

$$
\begin{gathered}
\mathcal{M} \frac{d}{d t} E+\frac{1}{3}(2 \mathcal{N}(H \diamond \bar{U})+H \diamond(\mathcal{N} \bar{U})+\bar{U} \diamond(\mathcal{N} H))=0, \\
\frac{d}{d t} \mathcal{M} \bar{U}+\frac{1}{3}\left(\mathcal{N}\left(\bar{U}^{2}\right)+\bar{U} \diamond(\mathcal{N} \bar{U})\right)+g \mathcal{N} E-\frac{1}{6}\left\{D ; \bar{U}_{t}\right\}=0,
\end{gathered}
$$


where we have introduced

$$
\left\{D ; \bar{U}_{t}\right\}=\mathcal{Q}\left(D^{2} \diamond \bar{U}_{t}\right)+D \diamond\left(\mathcal{Q}\left(D \diamond \bar{U}_{t}\right)\right)+2\left(D \diamond \bar{U}_{t}\right) \diamond(\mathcal{Q} D)-\bar{U}_{t} \diamond\left(\mathcal{Q} D^{2}\right)
$$

Equations (11)-(12) will be taken in the sequel as the classical scheme for the Peregrine equations and be used in Sections 5 and 6 to make some comparison with the new scheme introduced in the next section.

\section{A new setting for deriving discrete asymptotic mod- els.}

\subsection{Semi-discretization of the 2D-Euler equations in non-dimensional form.}

The aim of this section is to derive an alternative set of discrete equations, possibly having improved characteristics w.r.t. (11)-(12), for example a better evaluation of the shoaling gradient phenomenon. For that purpose, we propose to discretize the 2D-Euler equations with respect to one direction, $x$ for example, and then to perform an asymptotic analysis on the resulting equation, similar to the one used to obtain the Peregrine equations (1). The Euler equations written in terms of velocity $(u, w)$, pressure $p$, constant density $\rho$ and vertical gravity acceleration $g$ reads :

$$
\begin{gathered}
u_{t}+u u_{x}+w u_{z}+\frac{p_{x}}{\rho}=0, \\
w_{t}+u w_{x}+w w_{z}+\frac{p_{z}}{\rho}+g=0, \\
u_{x}+w_{z}=0 \\
u_{z}-w_{x}=0
\end{gathered}
$$

where the last equation represents the irrotationality condition. In this paper, since our aim is to obtain a new scheme for the Peregrine system, we restrict ourselves to the $2 \mathrm{D}$ version of the Euler equation. We deal with periodic boundary conditions in the $x$ direction, while on the free surface and sea-bed level we use the classical conditions :

- at the free surface $z=\eta$

$$
\begin{gathered}
w=\eta_{t}+u \eta_{x}, \\
p=0,
\end{gathered}
$$

- on the seafloor $z=-d$

$$
w=-u d_{x}
$$

Let $d_{0}$ be the averaged depth, $a$ a typical wave amplitude, and $\lambda$ a typical wavelength. The following usual non-dimensional variables are introduced

$$
\begin{gathered}
\tilde{x}=\frac{x}{\lambda}, \tilde{z}=\frac{z}{d_{0}}, \tilde{t}=\frac{\sqrt{g d_{0}}}{\lambda} t, \tilde{\eta}=\frac{\eta}{a}, \\
\tilde{u}=\frac{d_{0}}{a \sqrt{g d_{0}}} u, \tilde{w}=\frac{\lambda}{a} \frac{1}{\sqrt{g d_{0}}} w, \tilde{p}=\frac{p}{g d_{0} \rho}, \Delta_{\tilde{x}}=\frac{\Delta_{x}}{\lambda} .
\end{gathered}
$$


Classically, we now consider the nonlinearity and dispersion parameters $\varepsilon$ and $\sigma$ defined by

$$
\varepsilon=\frac{a}{d_{0}}, \sigma=\frac{d_{0}}{\lambda} .
$$

Using the notation introduced above, the Euler equations and the irrotationality condition can be recast in a non-dimensional form as

$$
\begin{gathered}
\varepsilon \tilde{u}_{\tilde{t}}+\varepsilon^{2} \tilde{u} \tilde{u}_{\tilde{x}}+\varepsilon^{2} \tilde{w} \tilde{u}_{\tilde{z}}+\tilde{p}_{\tilde{x}}=0, \\
\varepsilon \sigma^{2} \tilde{w}_{\tilde{t}}+\varepsilon^{2} \sigma^{2} \tilde{u} \tilde{w}_{\tilde{x}}+\varepsilon^{2} \sigma^{2} \tilde{w}_{\tilde{w}}+\tilde{p}_{\tilde{z}}+1=0, \\
\tilde{u}_{\tilde{x}}+\tilde{w}_{\tilde{z}}=0, \\
\tilde{u}_{\tilde{z}}-\sigma^{2} \tilde{w}_{\tilde{x}}=0 \quad\left(\text { so } \tilde{u}_{\tilde{z}}=\mathcal{O}\left(\sigma^{2}\right)\right),
\end{gathered}
$$

The boundary conditions become :

- at the free surface $\tilde{z}=\varepsilon \tilde{\eta}$

$$
\begin{gathered}
\tilde{w}=\tilde{\eta}_{\tilde{t}}+\varepsilon \tilde{u} \tilde{\eta}_{\tilde{x}}, \\
\tilde{p}=0,
\end{gathered}
$$

- at the bed $\tilde{z}=-\tilde{d}$

$$
\tilde{w}=-\tilde{d}_{\tilde{x}} \tilde{u}
$$

Our goal is to obtain a Boussinesq's type approximation of the Euler system (21)-(27), under the assumptions $\varepsilon<<1, \sigma<<1$, and in the specific regime $\varepsilon=\mathcal{O}\left(\sigma^{2}\right)$, meaning that there exists constant $C>0$ such that $\varepsilon \leq C \sigma^{2}$. Using (24), we obtain from (21)

$$
\varepsilon \tilde{u}_{\tilde{t}}+\varepsilon^{2} \tilde{u} \tilde{u}_{\tilde{x}}+\tilde{p}_{\tilde{x}}=-\varepsilon^{2} \sigma^{2} \tilde{w} \tilde{w}_{\tilde{x}} .
$$

We now apply a Galerkin method on the variable $x$ keeping $t$ and $z$ unchanged. It is assumed that the number of mesh points per wavelength is small, in particular $\Delta_{\tilde{x}}<1$, however, we assume $\Delta_{\tilde{x}}=\mathcal{O}(\sigma)$ (it transpires that $\Delta_{x}=\mathcal{O}\left(d_{0}\right)$ ).

In the sequel we drop the " $\sim$ " and we introduce for all $i \in\{0, \ldots, N\}, u_{i}(t, z)=$ $u\left(t, x_{i}, z\right), w_{i}(t, z)=w\left(t, x_{i}, z\right), \eta_{i}(t, z)=\eta\left(t, x_{i}, z\right), p_{i}(t, z)=p\left(t, x_{i}, z\right)$. In addition, the discrete horizontal velocity, wave height, depth, vertical velocity and pressure polynomials are written in the Galerkin basis as follows

$$
\begin{aligned}
& u_{\Delta}(t, x, z)=\sum_{j=0}^{N} u_{j}(t, z) \varphi_{j}(x), w_{\Delta}(t, x, z)=\sum_{j=0}^{N} w_{j}(t, z) \varphi_{j}(x), \eta_{\Delta}(t, x)=\sum_{j=0}^{N} \eta_{j}(t) \varphi_{j}(x), \\
& p_{\Delta}(t, x, z)=\sum_{j=0}^{N} p_{j}(t, z) \varphi_{j}(x), d_{\Delta}(x)=\sum_{j=0}^{N} d_{j} \varphi_{j}(x) .
\end{aligned}
$$

As in Section 3, we focus on periodic boundary condition that is we introduce $x_{-1}=x_{N}$ and $x_{N+1}=x_{0}$. The finite element discrete equations corresponding to (28)-(22)-(23)-(24) can be written as, for all $i \in\{0, \ldots, N\}$

$\mathrm{RR} \mathrm{n}^{\circ} 8806$

$$
\begin{aligned}
\varepsilon \frac{\Delta_{x}}{6} \frac{d}{d t}\left(u_{i+1}+4 u_{i}+u_{i-1}\right) & +\frac{\varepsilon^{2}}{3}\left(\frac{u_{i+1}^{2}-u_{i-1}^{2}}{2}+u_{i} \frac{u_{i+1}-u_{i-1}}{2}\right)+\frac{p_{i+1}-p_{i-1}}{2} \\
& =-\frac{\varepsilon^{2} \sigma^{2}}{3}\left(\frac{w_{i+1}^{2}-w_{i-1}^{2}}{2}+w_{i} \frac{w_{i+1}-w_{i-1}}{2}\right),
\end{aligned}
$$




$$
\begin{aligned}
& \varepsilon \sigma^{2} \frac{\Delta_{x}}{6} \frac{d}{d t}\left(w_{i+1}+4 w_{i}+w_{i-1}\right)+\frac{\Delta_{x}}{6} \frac{d}{d z}\left(p_{i+1}+4 p_{i}+p_{i-1}\right)+\Delta_{x} \\
&=-\varepsilon^{2} \sigma^{2}\left(u_{i} \frac{w_{i+1}-w_{i-1}}{2}-w_{i} \frac{u_{i+1}-u_{i-1}}{2}\right) \\
& \frac{u_{i+1}-u_{i-1}}{2}+ \frac{\Delta_{x}}{6} \frac{d}{d z}\left(w_{i+1}+4 w_{i}+w_{i-1}\right)=0 \\
& \frac{\Delta_{x}}{6} \frac{d}{d z}\left(u_{i+1}+4 u_{i}+u_{i-1}\right)-\sigma^{2} \frac{w_{i+1}-w_{i-1}}{2}=0
\end{aligned}
$$

For the boundary conditions, we propose to integrate (25)-(26) along the curve $z=\varepsilon \eta$ and equation (27) along the curve $z=-d$. For that purpose, we choose to introduce

$$
\begin{aligned}
\hat{w}_{\Delta} & =\sum_{i=0}^{N} w\left(t, \varepsilon \eta\left(t, x_{i}\right)\right) \phi_{i}(x), \check{w}_{\Delta}=\sum_{i=0}^{N} w\left(t,-d\left(x_{i}\right)\right) \phi_{i}(x), \\
\hat{u}_{\Delta} & =\sum_{i=0}^{N} u\left(t, \varepsilon \eta\left(t, x_{i}\right)\right) \phi_{i}(x), \check{u}_{\Delta}=\sum_{i=0}^{N} u\left(t,-d\left(x_{i}\right)\right) \phi_{i}(x) .
\end{aligned}
$$

The boundary conditions (25)-(26)-(27) then write

- at the free surface

$$
\begin{aligned}
& \frac{\Delta_{x}}{6}\left(w_{i+1}\left(t, \varepsilon \eta_{i+1}\right)+4 w_{i}\left(t, \varepsilon \eta_{i}\right)+w_{i-1}\left(t, \varepsilon \eta_{i-1}\right)\right)= \\
& \frac{\Delta_{x}}{6} \frac{d}{d t}\left(\eta_{i+1}(t)+4 \eta_{i}(t)+\eta_{i-1}(t)+\frac{1}{3}\left[\frac{\varepsilon \eta_{i+1}(t) u_{i+1}\left(t, \varepsilon \eta_{i+1}\right)-\varepsilon \eta_{i-1}(t) u_{i-1}\left(t, \varepsilon \eta_{i-1}\right)}{2}\right.\right. \\
& \left.-\varepsilon \eta_{i}(t) \frac{u_{i+1}\left(t, \varepsilon \eta_{i+1}\right)-u_{i-1}\left(t, \varepsilon \eta_{i-1}\right)}{2}+2 u_{i}\left(t, \varepsilon \eta_{i}\right) \frac{\varepsilon \eta_{i+1}(t)-\varepsilon \eta_{i-1}(t)}{2}\right] \\
& \frac{\Delta_{x}}{6}\left(p_{i+1}\left(t, \varepsilon \eta_{i+1}\right)+4 p_{i}\left(t, \varepsilon \eta_{i}\right)+p_{i-1}\left(t, \varepsilon \eta_{i-1}\right)\right)=0
\end{aligned}
$$

- at the bed

$$
\begin{aligned}
\frac{\Delta_{x}}{6}\left(w_{i+1}\left(t,-d_{i+1}\right)+4 w_{i}\left(t,-d_{i}\right)+w_{i-1}\left(t,-d_{i-1}\right)\right) & = \\
-\frac{1}{3}\left[\frac{d_{i+1} u_{i+1}\left(t,-d_{i+1}\right)-d_{i-1} u_{i-1}\left(t,-d_{i-1}\right)}{2}-d_{i} \frac{u_{i+1}\left(t,-d_{i+1}\right)-u_{i-1}\left(t,-d_{i-1}\right)}{2}\right. & \left.+2 u_{i}\left(t,-d_{i}\right) \frac{d_{i+1}-d_{i-1}}{2}\right] .
\end{aligned}
$$

Introducing the following column vector

$$
\begin{aligned}
& W=\left(w_{i}\right)_{0 \leq i \leq N}, U=\left(u_{i}\right)_{0 \leq i \leq N}, E=\left(\eta_{i}\right)_{0 \leq i \leq N}, P=\left(p_{i}\right)_{0 \leq i \leq N}, \mathcal{I}=\left(\begin{array}{c}
1 \\
\vdots \\
1
\end{array}\right) \\
& \hat{W}=\left(w_{i}\left(\varepsilon \eta_{i}\right)\right)_{0 \leq i \leq N}, \hat{U}=\left(u_{i}\left(\varepsilon \eta_{i}\right)\right)_{0 \leq i \leq N}, \check{W}=\left(w_{i}\left(-d_{i}\right)\right)_{0 \leq i \leq N}, \check{U}=\left(u_{i}\left(-d_{i}\right)\right)_{\text {I hima } \underline{\alpha} i \leq N},
\end{aligned}
$$


we can rewrite Equations (30)-(37) into the following matrix-form :

$$
\begin{gathered}
\varepsilon \frac{d}{d t} \mathcal{M} U+\frac{\varepsilon^{2}}{3}\left(\mathcal{N}\left(U^{2}\right)+U \diamond(\mathcal{N} U)\right)+\mathcal{N} P=-\frac{\varepsilon^{2} \sigma^{2}}{3}\left(\mathcal{N}\left(W^{2}\right)+W \diamond(\mathcal{N} W)\right) \\
\varepsilon \sigma^{2} \frac{d}{d t} \mathcal{M} W+\frac{d}{d z} \mathcal{M} P+\mathcal{I}=-\varepsilon^{2} \sigma^{2}(U \diamond(\mathcal{N} W)-W \diamond(\mathcal{N} U)) \\
\mathcal{N} U+\mathcal{M} \frac{d}{d z} W=0 \\
\mathcal{M} \frac{d}{d z} U-\sigma^{2} \mathcal{N} W=0
\end{gathered}
$$

The boundary conditions become

- at the free surface

$$
\begin{gathered}
\mathcal{M} \hat{W}=\frac{d}{d t} \mathcal{M} E+\frac{\varepsilon}{3}[\mathcal{N}(E \diamond \hat{U})-E \diamond(\mathcal{N} \hat{U})+2 \hat{U} \diamond(\mathcal{N} E)], \\
\mathcal{M} \hat{P}=0,
\end{gathered}
$$

- at the bottom

$$
\mathcal{M} \check{W}=-\frac{1}{3}[\mathcal{N}(D \diamond \check{U})-D \diamond(\mathcal{N} \check{U})+2 \check{U} \diamond(\mathcal{N} D)]
$$

System (38)-(44) represents the first step in our analysis. The next two sections are dedicated to the transformation of this system into an asymptotic set of equations.

\subsection{Asymptotic expansions on the velocity $U$ and the pressure $p$.}

In this section, we derive an asymptotic expansion in terms of $\sigma$ for the semi-discrete horizontal velocity $U=U(t, z)$ following the procedure presented by Peregrine in [15]. More precisely, we prove the following proposition.

Proposition 1. The pressure $p$ and the velocity $U$ satisfy expansion of the form

$$
\begin{gathered}
P=\varepsilon E-z \mathcal{I}+\varepsilon \sigma^{2}\left(\frac{z^{2}}{2} \mathcal{K} U^{0}+z\left[D ; U^{0}\right]\right)+\mathcal{O}\left(\varepsilon^{2} \sigma^{2}, \varepsilon \sigma^{4}\right) \\
U=\bar{U}+\sigma^{2}\left[\frac{D^{2}}{6} \diamond\left(\mathcal{K}^{2} \bar{U}\right)-\frac{z^{2}}{2} \mathcal{K}^{2} \bar{U}-z \mathcal{K}[D ; \bar{U}]-\frac{D}{2} \diamond(\mathcal{K}[D ; \bar{U}])\right]+\mathcal{O}\left(\varepsilon \sigma^{2}, \sigma^{4}\right),
\end{gathered}
$$

where the averaged velocity is defined in (56).

Proof. Since $\mathcal{M}$ is invertible, we obtain from the integration of (41) between 0 and an arbitrary depth $z$,

$$
U(t, z)=U^{0}(t)+\mathcal{O}\left(\sigma^{2}\right),
$$

where $U^{0}(t)$ is a constant depending only on $t$ and corresponds to the value of $U$ at $z=0$. We directly deduce that

$$
\check{U}(t)=U^{0}(t)+\mathcal{O}\left(\sigma^{2}\right) .
$$

Substituting relation (45) in equation (40) and setting $\mathcal{K}=\mathcal{M}^{-1} \mathcal{N}$, we derive

$$
\frac{d}{d z} W=-\mathcal{K} U^{0}+\mathcal{O}\left(\sigma^{2}\right)
$$


Integrating each line $i \in\{0, \ldots, N\}$ of equation (47) with respect to $z$ between $-d_{i}$ and an arbitrary depth $z\left(-d_{i}<z<\epsilon \eta_{i}\right)$, using the boundary condition (44) and the estimates (45) on $U$ and (46) on $\check{U}$, we obtain

$$
\begin{aligned}
W= & -z \mathcal{K} U^{0}-D \diamond\left(\mathcal{K} U^{0}\right)-\frac{1}{3}\left[\mathcal{K}\left(D \diamond U^{0}\right)-\mathcal{M}^{-1}\left(D \diamond\left(\mathcal{N} U^{0}\right)\right)\right. \\
& \left.+2 \mathcal{M}^{-1}\left(U^{0} \diamond(\mathcal{N} D)\right)\right]+\mathcal{O}\left(\sigma^{2}\right) .
\end{aligned}
$$

Plugging (48) in (41) and integrating the resulting equation between 0 and $z$, one derives the following expansion on $U$

$$
\begin{aligned}
U= & U^{0}-\sigma^{2}\left(\frac{z^{2}}{2} \mathcal{K}^{2} U^{0}+z \mathcal{K}\left(D \diamond\left(\mathcal{K} U^{0}\right)\right)+\frac{z}{3} \mathcal{K}\left[\mathcal{K}\left(D \diamond U^{0}\right)\right.\right. \\
& \left.\left.-\mathcal{M}^{-1}\left(D \diamond\left(\mathcal{N} U^{0}\right)\right)+2 \mathcal{M}^{-1}\left(U^{0} \diamond(\mathcal{N} D)\right)\right]\right)+\mathcal{O}\left(\sigma^{4}\right)
\end{aligned}
$$

Looking for a similar expansion on the pressure array $P$, we substitute Equation (48) in Equation (39) to obtain

$$
\begin{aligned}
\frac{d}{d z} \mathcal{M} P= & -\mathcal{I}-\varepsilon \sigma^{2} \frac{d}{d t}\left(z \mathcal{K} U^{0}+\mathcal{M}\left(D \diamond\left[\mathcal{K} U^{0}\right]\right)\right. \\
& \left.+\frac{1}{3}\left[\mathcal{N}\left(D \diamond U^{0}\right)-D \diamond\left[\mathcal{N} U^{0}\right]+2 U^{0} \diamond[\mathcal{N} D]\right]\right)+\mathcal{O}\left(\varepsilon^{2} \sigma^{2}, \varepsilon \sigma^{4}\right)
\end{aligned}
$$

Using the fact that

$$
\mathcal{M I}=\mathcal{I}
$$

the previous relation becomes

$$
\begin{aligned}
\frac{d}{d z} P= & -\mathcal{I}-\varepsilon \sigma^{2} \frac{d}{d t}\left(z \mathcal{K} U^{0}+D \diamond\left[\mathcal{K} U^{0}\right]\right. \\
& \left.+\frac{1}{3}\left[\mathcal{K}\left(D \diamond U^{0}\right)-\mathcal{M}^{-1}\left(D \diamond\left(\mathcal{N} U^{0}\right)\right)+2 \mathcal{M}^{-1}\left(U^{0} \diamond(\mathcal{N} D)\right)\right]\right)+\mathcal{O}\left(\varepsilon^{2} \sigma^{2}, \varepsilon \sigma^{4}\right) .
\end{aligned}
$$

In view of (51), it is natural to introduce the following bracket

$$
[A ; B]=A \diamond(\mathcal{K} B)+\frac{1}{3}\left[\mathcal{K}(A \diamond B)-\mathcal{M}^{-1}(A \diamond(\mathcal{N} B))+2 \mathcal{M}^{-1}(B \diamond(\mathcal{N} A))\right]
$$

Remark that (49) then becomes

$$
U=U^{0}-\sigma^{2}\left(\frac{z^{2}}{2} \mathcal{K}^{2} U^{0}+z\left[D ; U^{0}\right]\right)+\mathcal{O}\left(\sigma^{4}\right) .
$$

Furthermore, integrating (51) with respect to $z$ from an arbitrary depth to the free surface $\varepsilon \eta_{i}$, we can write

$$
P=\varepsilon E-z \mathcal{I}+\varepsilon \sigma^{2}\left(\frac{z^{2}}{2} \mathcal{K} U^{0}+z\left[D ; U^{0}\right]\right)+\mathcal{O}\left(\varepsilon^{2} \sigma^{2}, \varepsilon \sigma^{4}\right)
$$

Substituting equations (54) and (49) in (38), we obtain an equation for the zero-th order velocity $U^{0}$, equivalent to Equation 2.28 in [17], which reads :

$$
\frac{d}{d t} \mathcal{M} U^{0}+\frac{\varepsilon}{3}\left(\mathcal{N}\left(U^{0} \diamond U^{0}\right)+U^{0} \diamond\left(\mathcal{N} U^{0}\right)\right)+\mathcal{N} E=\mathcal{O}\left(\varepsilon \sigma^{2}, \sigma^{4}\right) .
$$


Note that the choice of the constant of integration in (45) is not unique. However it transpires that the choice of $U^{0}$ (which is the value of the horizontal velocity $U$ at $z=0$ ) is not optimal as observed in [17]. This is why, in the sequel, we are going to get rid of it by introducing the averaged velocity matrix $\bar{U}=\left(\bar{u}_{i}\right)_{0 \leq i \leq N}$ where

$$
\bar{u}_{i}=\frac{1}{d_{i}+\varepsilon \eta_{i}} \int_{-d_{i}}^{\varepsilon \eta_{i}} u_{i} d z
$$

and by looking for the equation satisfied by $\bar{U}$. In this direction, we first derive the relation between $U_{0}$ and $\bar{U}$. Equation (53) provides, for all $i \in\{0, \ldots, N\}$,

$$
u_{i}=u_{i}^{0}-\sigma^{2}\left[\frac{z^{2}}{2}\left(\mathcal{K}^{2} U^{0}\right)_{i}+z\left(\mathcal{K}\left[D ; U^{0}\right]\right)_{i}\right]+\mathcal{O}\left(\sigma^{4}\right)
$$

and by integration between $-d_{i}$ and $\varepsilon \eta_{i}$, we immediately get, using Taylor expansion,

$$
\begin{aligned}
\bar{u}_{i} & =u_{i}^{0}-\frac{1}{\varepsilon \eta_{i}+d_{i}} \sigma^{2}\left[\int_{-d_{i}}^{\varepsilon \eta_{i}} \frac{z^{2}}{2} d z\left(\mathcal{K}^{2} U^{0}\right)_{i}+\int_{-d_{i}}^{\varepsilon \eta_{i}} z d z\left(\mathcal{K}\left[D ; U^{0}\right]\right)_{i}\right]+\mathcal{O}\left(\sigma^{4}\right), \\
& =u_{i}^{0}-\frac{\sigma^{2}}{\left(d_{i}+\varepsilon \eta_{i}\right)}\left[\frac{d_{i}^{3}}{6}\left(\mathcal{K}^{2} U^{0}\right)_{i}-\frac{d_{i}^{2}}{2}\left(\mathcal{K}\left[D ; U^{0}\right]\right)_{i}\right]+\mathcal{O}\left(\varepsilon \sigma^{2}, \sigma^{4}\right), \\
& =u_{i}^{0}-\sigma^{2}\left[\frac{d_{i}^{2}}{6}\left(\mathcal{K}^{2} U^{0}\right)_{i}-\frac{d_{i}}{2}\left(\mathcal{K}\left[D ; U^{0}\right]\right)_{i}\right]+\mathcal{O}\left(\varepsilon \sigma^{2}, \sigma^{4}\right) .
\end{aligned}
$$

This furnishes the desired relation

$$
\bar{U}=U^{0}-\sigma^{2}\left[\frac{D^{2}}{6} \diamond\left(\mathcal{K}^{2} U^{0}\right)-\frac{D}{2} \diamond\left(\mathcal{K}\left[D ; U^{0}\right]\right)\right]+\mathcal{O}\left(\varepsilon \sigma^{2}, \sigma^{4}\right),
$$

or equivalently

$$
U^{0}=\bar{U}+\sigma^{2}\left[\frac{D^{2}}{6} \diamond\left(\mathcal{K}^{2} U^{0}\right)-\frac{D}{2} \diamond\left(\mathcal{K}\left[D ; U^{0}\right]\right)\right]+\mathcal{O}\left(\varepsilon \sigma^{2}, \sigma^{4}\right) .
$$

Then it transpires that

$$
U^{0}=\bar{U}+\mathcal{O}\left(\varepsilon, \sigma^{2}\right)
$$

Substituting (59) in (58), we derive

$$
U^{0}=\bar{U}+\sigma^{2}\left[\frac{D^{2}}{6} \diamond\left(\mathcal{K}^{2} \bar{U}\right)-\frac{D}{2} \diamond(\mathcal{K}[D ; \bar{U}])\right]+\mathcal{O}\left(\varepsilon \sigma^{2}, \sigma^{4}\right) .
$$

Finally, plugging (60) into (53), one obtains the expansion of $U$ as a function of the depth averaged velocity $\bar{U}$

$$
U=\bar{U}+\sigma^{2}\left[\frac{D^{2}}{6} \diamond\left(\mathcal{K}^{2} \bar{U}\right)-\frac{z^{2}}{2} \mathcal{K}^{2} \bar{U}-z \mathcal{K}[D ; \bar{U}]-\frac{D}{2} \diamond(\mathcal{K}[D ; \bar{U}])\right]+\mathcal{O}\left(\varepsilon \sigma^{2}, \sigma^{4}\right),
$$

which ends the proof of Proposition 1.

\subsection{Depth-averaged equations}

The aim of this section is to provide the final new discrete numerical model of Peregrine's type. In order to derive the equation on $\bar{U}$ (known as the momentum equation in the literature), we substitute (60) in (55) to obtain :

$$
\begin{aligned}
& \frac{d}{d t} \mathcal{M} \bar{U}+\frac{\varepsilon}{3}\left[\mathcal{N}\left(\bar{U}^{2}\right)+\bar{U} \diamond(\mathcal{N} \bar{U})\right]+\mathcal{N} E \\
& +\sigma^{2} \mathcal{M} \frac{d}{d t}\left[\frac{D^{2}}{6} \diamond\left(\mathcal{K}^{2} \bar{U}\right)-\frac{D}{2} \diamond \mathcal{K}[D ; \bar{U}]\right]=\mathcal{O}\left(\varepsilon \sigma^{2}, \sigma^{4}\right)
\end{aligned}
$$


In addition, to derive an equation on $E$ (that is the continuity equation), we combine (42) and (44) to get

$$
\begin{aligned}
\hat{W}-\check{W}=\frac{d}{d t} E+ & \varepsilon \frac{\mathcal{M}^{-1}}{3}[\mathcal{N}(E \diamond \hat{U})-E \diamond(\mathcal{N} \hat{U})+2 \hat{U} \diamond(\mathcal{N} E)] \\
& +\frac{\mathcal{M}^{-1}}{3}[\mathcal{N}(D \diamond \check{U})-D \diamond(\mathcal{N} \check{U})+2 \check{U} \diamond(\mathcal{N} D)] .
\end{aligned}
$$

We integrate each lines of $(63)$ between $-d_{i}$ and $\varepsilon \eta_{i}$, for all $i \in\{0, \ldots, N\}$, to obtain

$$
\int_{-d_{i}}^{\varepsilon \eta_{i}}(\mathcal{K} U)_{i} d z+\hat{W}_{i}-\check{W}_{i}=0,
$$

which can be recast as

$$
E_{t}+[H ; \bar{U}]+B=0
$$

where

$$
\begin{aligned}
B & =\left(\int_{-d_{i}}^{\varepsilon \eta_{i}}(\mathcal{K} U)_{i} d z\right)_{0 \leq i \leq N}-[H ; \bar{U}] \\
& +\frac{\varepsilon}{3}\left[\mathcal{K}(E \diamond \hat{U})-\mathcal{M}^{-1}(E \diamond(\mathcal{N} \hat{U}))+2 \mathcal{M}^{-1}(\hat{U} \diamond(\mathcal{N} E))\right] \\
& -\frac{1}{3}\left[\mathcal{K}(-D \diamond \check{U})-\mathcal{M}^{-1}(-D \diamond(\mathcal{N} \check{U}))+2 \mathcal{M}^{-1}(\check{U} \diamond(\mathcal{N}(-D)))\right] .
\end{aligned}
$$

We can remark that the expression $B$ is no more than a discretized version of the socalled Leibniz' Rule ${ }^{1}$. As a consequence, it transpires that $B$ has the same accuracy of order $\mathcal{O}\left(\varepsilon \sigma^{2}, \sigma^{4}\right)$ than that of the equations and then can be neglected in the sequel (see Proposition 2 below for more details). In order to be more precise, we compute explicitly $B$ by taking successively $z=\varepsilon \eta_{i}$ and $z=-d_{i}$ in (61) to obtain the values of $\hat{U}$ and $\check{U}$ :

$$
\begin{aligned}
& \hat{U}=\bar{U}+\sigma^{2}\left[\frac{D^{2}}{6} \diamond\left(\mathcal{K}^{2} \bar{U}\right)-\frac{\varepsilon^{3} E^{2}}{2} \mathcal{K}^{2} \bar{U}-\varepsilon E \diamond \mathcal{K}[D ; \bar{U}]-\frac{D}{2} \diamond(\mathcal{K}[D ; \bar{U}])\right]+\mathcal{O}\left(\varepsilon \sigma^{2}, \sigma^{4}\right) \\
& \check{U}=\bar{U}+\sigma^{2}\left[-\frac{D^{2}}{3} \diamond\left(\mathcal{K}^{2} \bar{U}\right)+\frac{D}{2} \diamond(\mathcal{K}[D ; \bar{U}])\right]+\mathcal{O}\left(\varepsilon \sigma^{2}, \sigma^{4}\right)
\end{aligned}
$$

By substituting Equations (61) and (66) into Equation (65), this provides the complete expression of $B$

$$
\begin{aligned}
& B=\sigma^{2}\left[\frac{1}{6} D \diamond\left(\mathcal{K}\left(D^{2} \diamond\left(\mathcal{K}^{2} \bar{U}\right)\right)\right)-\frac{1}{6} D^{3} \diamond\left(\mathcal{K}^{3} \bar{U}\right)-\frac{1}{9} \mathcal{K}\left(D^{3} \diamond\left(\mathcal{K}^{2} \bar{U}\right)\right)\right. \\
& +\frac{1}{9} \mathcal{M}^{-1} D \diamond\left(\mathcal{N}\left(D^{2} \diamond\left(\mathcal{K}^{2} \bar{U}\right)\right)\right)+\frac{1}{2} D^{2} \diamond\left(\mathcal{K}^{2}[D ; \bar{U}]\right)-\frac{1}{2} D \diamond(\mathcal{K}(D \diamond(\mathcal{K}[D ; \bar{U}]))) \\
& +\frac{1}{6} \mathcal{K}\left(D^{2} \diamond(\mathcal{K}[D ; \bar{U}])\right)-\frac{1}{6} \mathcal{M}^{-1} D \diamond(\mathcal{N} D \diamond(\mathcal{K}[D ; \bar{U}])) \\
& \left.-\frac{2}{9} \mathcal{M}^{-1}(\mathcal{N} D) \diamond\left(D^{2} \diamond\left(\mathcal{K}^{2} \bar{U}\right)\right)+\frac{1}{3} \mathcal{M}^{-1}((\mathcal{N} D) \diamond(D \diamond(\mathcal{K}[D ; \bar{U}])))\right]+\mathcal{O}\left(\varepsilon \sigma^{2}, \sigma^{4}\right) .
\end{aligned}
$$

\footnotetext{
${ }^{1}$ We recall the Leibniz' Rule: Given $f(x, z), a(x)$ and $b(x)$, where $f$ and $\frac{\partial f}{\partial x}$ are continuous in $x$ and $z$, and $a$ and $b$ are differentialble functions of $x$,$$
\frac{\partial}{\partial x}\left(\int_{a(x)}^{b(x)} f(x, z) d z\right)=\int_{a(x)}^{b(x)} \frac{\partial}{\partial x} f(x, z) d z+f(x, b(x)) b^{\prime}(x)-f(x, a(x)) a^{\prime}(x) .
$$ 
Finally, our new non-dimensionalized system reads (note that we have multiply (64) by $\mathcal{M})$

$$
\begin{gathered}
\frac{d}{d t} \mathcal{M} E+\mathcal{M}[H ; \bar{U}]+\mathcal{M} B=0 . \\
\frac{d}{d t} \mathcal{M} \bar{U}+\frac{\varepsilon}{3}\left[\mathcal{N}\left(\bar{U}^{2}\right)+\bar{U} \diamond(\mathcal{N} \bar{U})\right]+\mathcal{N} E \\
+\sigma^{2} \mathcal{M} \frac{d}{d t}\left[\frac{D^{2}}{6} \diamond\left(\mathcal{K}^{2} \bar{U}\right)-\frac{D}{2} \diamond \mathcal{K}[D ; \bar{U}]\right]=\mathcal{O}\left(\varepsilon \sigma^{2}, \sigma^{4}\right)
\end{gathered}
$$

To go further, we now investigate the behavior of the vector $B$ by establishing the following proposition.

Proposition 2. For any bathymetry d, the additional term B in Equation (67) satisfies

$$
B=\mathcal{O}\left(\varepsilon \sigma^{2}, \sigma^{4}\right) .
$$

As a consequence, the numerical scheme (67)-(68) becomes

$$
\begin{gathered}
\frac{d}{d t} \mathcal{M} E+\mathcal{M}[H ; \bar{U}]=\mathcal{O}\left(\varepsilon \sigma^{2}, \sigma^{4}\right), \\
\frac{d}{d t} \mathcal{M} \bar{U}+\frac{1}{3}\left[\mathcal{N}\left(\bar{U}^{2}\right)+\bar{U} \diamond(\mathcal{N} \bar{U})\right]+\mathcal{N} E-\sigma^{2} \frac{d}{d t}\left[\frac{d_{0}^{2}}{3}(\mathcal{N} \mathcal{K} \bar{U})\right]=\mathcal{O}\left(\varepsilon \sigma^{2}, \sigma^{4}\right),
\end{gathered}
$$

and is consistent with the Peregrine Equations (1).

Proof. For a better understanding, we first assume that the batymetry $d=d_{0}$ is constant. In this setting, one has $D=d_{0} \mathcal{I}$ and the operator $D \diamond$ is no more than the multiplication by the real $d_{0}$, that is, for example, $D \diamond U=d_{0} U$. Hence $B$ is equal to

$$
\begin{aligned}
B & =\sigma^{2}\left[\frac{d_{0}^{3}}{6} \mathcal{K}^{3} \bar{U}-\frac{d_{0}^{3}}{6} \mathcal{K}^{3} \bar{U}-\frac{d_{0}^{3}}{9} \mathcal{K}^{3} \bar{U}+\frac{d_{0}^{3}}{9} \mathcal{K}^{3} \bar{U}+\frac{d_{0}^{3}}{2} \mathcal{K}^{3} \bar{U}-\frac{d_{0}^{3}}{2} \mathcal{K}^{3} \bar{U}+\frac{d_{0}^{3}}{6} \mathcal{K}^{3} \bar{U}-\frac{d_{0}^{3}}{6} \mathcal{K}^{3} \bar{U}\right] \\
& +\mathcal{O}\left(\varepsilon \sigma^{2}, \sigma^{4}\right) . \\
& =0+\mathcal{O}\left(\varepsilon \sigma^{2}, \sigma^{4}\right) .
\end{aligned}
$$

More generally, assume now that the bathymetry $d$ is not constant. For any regular function $u$ and its discrete version $\left(u_{i}\right)_{0 \leq i \leq N}$, a Taylor expansion provides

$$
u_{i+1}=u_{i}+\Delta_{x} u_{x}\left(x_{i}\right)+\frac{\Delta_{x}^{2}}{2} u_{x x}\left(x_{i}\right)+\frac{\Delta_{x}^{3}}{6} u_{x x x}\left(x_{i}\right)+\ldots,
$$

and

$$
u_{i-1}=u_{i}-\Delta_{x} u_{x}\left(x_{i}\right)+\frac{\Delta_{x}^{2}}{2} u_{x x}\left(x_{i}\right)-\frac{\Delta_{x}^{3}}{6} u_{x x x}\left(x_{i}\right)+\ldots
$$

Combining (71) and (72), we can prove that, for all $i \in\{0, \ldots, N\}$

$$
\begin{gathered}
(\mathcal{N} U)_{i}=u_{x}\left(x_{i}\right)+\frac{\Delta_{x}^{2}}{6} u_{x x}\left(x_{i}\right)+\mathcal{O}\left(\Delta_{x}^{4}\right), \\
(\mathcal{K} U)_{i}=u_{x}\left(x_{i}\right)+\mathcal{O}\left(\Delta_{x}^{4}\right), \\
\left(\mathcal{M}^{-1} U\right)_{i}=u\left(x_{i}\right)-\frac{\Delta_{x}^{2}}{6} u_{x x}\left(x_{i}\right)+\mathcal{O}\left(\Delta_{x}^{4}\right) .
\end{gathered}
$$

$\mathrm{RR} \mathrm{n}^{\circ} 8806$ 
Plugging these expansions in Equations (67) and (68), we obtain, dividing by $\Delta_{x}$ at each point $x_{i}$

$$
\begin{gathered}
\eta_{t}+[h \bar{u}]_{x}-\frac{\Delta_{x}^{2}}{6}\left[\eta_{t x x}+\left[h \bar{u}_{x x x}+h_{x x} \bar{u}_{x}+\sigma^{2}\left(\frac{d^{2} d_{x x} \bar{u}_{x x x}}{6}+\frac{7}{6} d d_{x} d_{x x} \bar{u}_{x x}\right.\right.\right. \\
\left.\left.+\left(\frac{3}{2} d d_{x x}^{2} d_{x}^{2} d_{x x}+\frac{5}{6} d d_{x} d_{x x x}\right) \bar{u}_{x}+d d_{x x} d_{x x x} \bar{u}\right)\right]+\mathcal{O}\left(\Delta_{x}^{4}\right)=\mathcal{O}\left(\varepsilon \sigma^{2}, \sigma^{4}\right), \\
\bar{u}_{t}+\bar{u} \bar{u}_{x}+\eta_{x}+\sigma^{2}\left(\frac{d^{2}}{6} \bar{u}_{t x x}-\frac{d}{2}[d \bar{u}]_{t x x}\right)+\frac{\Delta_{x}^{2}}{6}\left[\left(\bar{u}_{t}+\bar{u} \bar{u}_{x}+\eta_{x}+\sigma^{2} \frac{d^{2}}{6} \bar{u}_{t x x}\right.\right. \\
\left.\left.-\sigma^{2} \frac{d}{2}[d \bar{u}]_{t x x}\right)_{x x}-\bar{u}_{x} \bar{u}_{x x}+\sigma^{2} \frac{d}{2}\left(d_{x x} \bar{u}_{x}\right)_{x}\right]+\mathcal{O}\left(\Delta_{x}^{4}\right)=\mathcal{O}\left(\varepsilon \sigma^{2}, \sigma^{4}\right),
\end{gathered}
$$

proving that our numerical scheme is consistent with the continuous Peregrine equations(1).

In addition, $B$ is equal to

$$
\begin{aligned}
B= & -\sigma^{2} \frac{\Delta_{x}^{2}}{6}\left(\frac{d^{2} d_{x x} \bar{u}_{x x x}}{6}+\frac{7}{6} d d_{x} d_{x x} \bar{u}_{x x}+\left(\frac{3}{2} d d_{x x}^{2} d_{x}^{2} d_{x x}+\frac{5}{6} d d_{x} d_{x x x}\right) \bar{u}_{x}+d d_{x x} d_{x x x} \bar{u}\right) \\
& +\mathcal{O}\left(\Delta_{x}^{4}\right)+\mathcal{O}\left(\varepsilon \sigma^{2}, \sigma^{4}\right),
\end{aligned}
$$

from which it transpires that $B$ contains only terms of order $\Delta_{x}^{2} \sigma^{2}, \varepsilon \sigma^{2}$ or $\sigma^{4}$ (actually, $B$ is consistent with Leibniz' Rule). Recalling that $\Delta_{\tilde{x}}=\mathcal{O}(\sigma)$, one has

$$
B=\sigma^{2} \mathcal{O}\left(\Delta_{\tilde{x}}^{2}\right)+\mathcal{O}\left(\varepsilon \sigma^{2}, \sigma^{4}\right)=\mathcal{O}\left(\varepsilon \sigma^{2}, \sigma^{4}\right),
$$

which ends the proof of Proposition 2

To end this section, we return to the physical variables and neglect the contribution of $B$ in (67)-(68) to obtain our new numerical scheme for the Peregrine Equations (1)

$$
\begin{gathered}
\frac{d}{d t} \mathcal{M} E+\mathcal{M}[H ; \bar{U}]=0 \\
\frac{d}{d t} \mathcal{M} \bar{U}+\frac{1}{3}\left[\mathcal{N}\left(\bar{U}^{2}\right)+\bar{U} \diamond(\mathcal{N} \bar{U})\right]+g \mathcal{N} E+\mathcal{M} \frac{d}{d t}\left[\frac{D^{2}}{6} \diamond\left(\mathcal{K}^{2} \bar{U}\right)-\frac{D}{2} \diamond(\mathcal{K}[D ; \bar{U}])\right]=0
\end{gathered}
$$

\section{Study of the linear dispersion characteristics.}

The aim of this section is to give some insights to measure the accuracy of the new method developped in the previous sections. For that purpose, we exhibit the dispersion relation as well as the shoaling coefficients of the linearized version of the scheme (73)-(74). This study is widely inspired by the one proposed by Dingemans in [6] in the context of slowlyvarying water depth, that is we assume that $d=d(\beta x)$ with $\beta<<1$. For the sake of completness, we also compare our computations with the ones performed on the linearized version of the classical scheme (11)-(12).

\subsection{Linear characteristics of the new numerical model.}

We first introduce the linearized version of the scheme (73)-(74) around the rest state which reads

$$
\frac{d}{d t} \mathcal{M} E+\mathcal{M}[D ; \bar{U}]=0
$$




$$
\frac{d}{d t} \mathcal{M} \bar{U}+g \mathcal{N} E+\mathcal{M} \frac{d}{d t}\left[\frac{D^{2}}{6} \diamond\left(\mathcal{K}^{2} \bar{U}\right)-\frac{D}{2} \diamond(\mathcal{K}[D ; \bar{U}])\right]=0 .
$$

As usual, when one deals with linear equations, a lot of computations can be performed explicitly. Indeed, differentiating (76) with respect to $t$, multiplying (75) by $\mathcal{N}$ and substituting the resulting equations, one obtains a decouple equation on the vector $\bar{U}$ :

$$
\mathcal{M} \bar{U}_{t t}-g \mathcal{N}[D ; \bar{U}]+\mathcal{M}\left[\frac{D^{2}}{6} \diamond\left(\mathcal{K}^{2} \bar{U}\right)-\frac{D}{2} \diamond(\mathcal{K}[D ; \bar{U}])\right]_{t t}=0 .
$$

In order to exhibit the dispersion relation associated with (75)-(76), we then look for a plane-wave solution under the form

$$
\bar{U}=\left(\bar{u}_{i}\right)_{0 \leq i \leq N}
$$

where

$$
\bar{u}_{i}=\bar{u}\left(t, x_{i}\right) \text { with } \bar{u}(t, x)=\mathbf{U}(\beta x) \exp \left[-j \omega t+\frac{j}{\beta} K(\beta x)\right], j^{2}=-1 .
$$

Owning the solution $\bar{U}$, it is pertinent to introduce the wave number

$$
k(\beta x)=\frac{\partial}{\partial x}\left(\frac{1}{\beta} K(\beta x)\right),
$$

and for all $i=0, \ldots, N, k_{i}=k\left(\beta x_{i}\right)$. Then, we determine conditions on $k$ and $\mathbf{U}$ so that $\bar{U}$ is a solution to the linear system (77). A Taylor expansion around the point $x=x_{i}$ provides directly

$$
\begin{aligned}
& \bar{u}_{i+1}=\left[1+\beta\left(j \frac{\Delta_{x}^{2}}{2} k^{\prime}\left(\beta x_{i}\right)+\Delta_{x} \frac{\mathbf{U}^{\prime}\left(\beta x_{i}\right)}{\mathbf{U}_{i}}\right)\right] \bar{u}_{i} e^{j k_{i} \Delta_{x}}+\mathcal{O}\left(\beta^{2}\right), \\
& \bar{u}_{i-1}=\left[1+\beta\left(j \frac{\Delta_{x}^{2}}{2} k^{\prime}\left(\beta x_{i}\right)-\Delta_{x} \frac{\mathbf{U}^{\prime}\left(\beta x_{i}\right)}{\mathbf{U}_{i}}\right)\right] \bar{u}_{i} e^{-j k_{i} \Delta_{x}}+\mathcal{O}\left(\beta^{2}\right) .
\end{aligned}
$$

In view of (77), we deduce that, $\forall i \in\{1, . ., n\}$

$$
\begin{aligned}
(\mathcal{N} \bar{U})_{i}= & \left(j k_{i} \sin _{c}\left(k_{i} \Delta_{x}\right)-\beta \frac{k_{i}^{2} \Delta_{x}^{2}}{2} \sin _{c}\left(k_{i} \Delta_{x}\right) \frac{k^{\prime}\left(\beta x_{i}\right)}{k_{i}}\right. \\
& \left.+\beta \cos \left(k_{i} \Delta_{x}\right) \frac{\mathbf{U}^{\prime}\left(\beta x_{i}\right)}{\mathbf{U}_{i}}\right) \bar{u}_{i}+\mathcal{O}\left(\beta^{2}\right) . \\
(\mathcal{M} \bar{U})_{i}= & \left(\frac{1}{3}\left(2+\cos \left(k_{i} \Delta_{x}\right)\right)+j \beta \frac{k_{i} \Delta_{x}^{2}}{6}\left(\cos \left(k_{i} \Delta_{x}\right) \frac{k^{\prime}\left(\beta x_{i}\right)}{k_{i}}+\right.\right. \\
& \left.\left.2 \sin _{c}\left(k_{i} \Delta_{x}\right) \frac{\mathbf{U}^{\prime}\left(\beta x_{i}\right)}{\mathbf{U}_{i}}\right)\right) \bar{u}_{i}+\mathcal{O}\left(\beta^{2}\right) .
\end{aligned}
$$

Note that it is not possible to plug directly (81)-(82) into (77), due to the presence of the vector $\left(\mathcal{M}^{-1} \bar{U}\right)$ in the bracket $[D ; \bar{U}]$. Indeed, it is necessary to express each term $\left(\mathcal{M}^{-1} \bar{U}\right)$ with respect to $\bar{u}_{i}$. To overcome this difficulty, the idea is to introduce the following new variables :

$$
\begin{aligned}
& V=\mathcal{M}^{-1} \mathcal{N}(D \diamond \bar{U}), X=\mathcal{M}^{-1} \mathcal{N} \bar{U}, Z=\mathcal{M}^{-1}(D \diamond(\mathcal{N} \bar{U})) \\
& W=\mathcal{M}^{-1}(\bar{U} \diamond(\mathcal{N} D)), Y=\mathcal{M}^{-1} \mathcal{N} X \\
& T=\left(D \diamond X+\frac{1}{3}(V-Z+2 W)\right), S=\mathcal{M}^{-1} \mathcal{N} T .
\end{aligned}
$$


and to perform asymptotic expansions of order $\beta^{2}$ on these variables. Using these new vectors, one can rewrite Equation (77) into

$$
\mathcal{M} \bar{U}_{t t}-g \mathcal{N} T+\mathcal{M}\left(\frac{D^{2}}{6} \diamond Y_{t t}-\frac{D}{2} \diamond S_{t t}\right)=0
$$

Note first that the vectors $V, X, Z, W, Y$ and $T$ depends only on $\bar{U}$. It is then natural to introduce the following functions

$$
\begin{gathered}
\mathbf{v}=\mathbf{V}(\beta x) \exp \left[-j \omega t+\frac{j}{\beta} K(\beta x)\right], \mathbf{x}=\mathbf{X}(\beta x) \exp \left[-j \omega t+\frac{j}{\beta} K(\beta x)\right], \\
\mathbf{z}=\mathbf{Z}(\beta x) \exp \left[-j \omega t+\frac{j}{\beta} K(\beta x)\right], \mathbf{w}=\mathbf{W}(\beta x) \exp \left[-j \omega t+\frac{j}{\beta} K(\beta x)\right], \\
\mathbf{y}=\mathbf{Y}(\beta x) \exp \left[-j \omega t+\frac{j}{\beta} K(\beta x)\right], \mathbf{t}=\mathbf{T}(\beta x) \exp \left[-j \omega t+\frac{j}{\beta} K(\beta x)\right], \\
\mathbf{s}=\mathbf{S}(\beta x) \exp \left[-j \omega t+\frac{j}{\beta} K(\beta x)\right],
\end{gathered}
$$

where $\mathbf{V}(\beta x), \mathbf{X}(\beta x), \mathbf{Z}(\beta x), \mathbf{W}(\beta x), \mathbf{Y}(\beta x), \mathbf{T}(\beta x)$ and $\mathbf{S}(\beta x)$ denote the amplitude of repectively $\mathbf{v}, \mathbf{x}, \mathbf{z}, \mathbf{w}, \mathbf{y}, \mathbf{t}$ and $\mathbf{s}$. Using Equations (81) and (82), one can rewite (83) for all $i \in\{1, \ldots, n\}$

$$
\begin{aligned}
& -\omega^{2}\left(\frac{1}{3}\left(2+\cos \left(k_{i} \Delta_{x}\right)\right)+j \beta \frac{k_{i} \Delta_{x}^{2}}{6}\left(\cos \left(k_{i} \Delta_{x}\right) \frac{k^{\prime}\left(\beta x_{i}\right)}{k_{i}}+2 \sin _{c}\left(k_{i} \Delta_{x}\right) \frac{\mathbf{U}^{\prime}\left(\beta x_{i}\right)}{\bar{u}_{i}}\right)\right) \bar{u}_{i} \\
& -g\left(j k_{i} \sin _{c}\left(k_{i} \Delta_{x}\right)-\beta \frac{k_{i}^{2} \Delta_{x}^{2}}{2} \sin _{c}\left(k_{i} \Delta_{x}\right) \frac{k^{\prime}\left(\beta x_{i}\right)}{k_{i}}+\beta \cos \left(k_{i} \Delta_{x}\right) \frac{\mathbf{T}^{\prime}\left(\beta x_{i}\right)}{\mathbf{T}\left(\beta x_{i}\right)}\right) \mathbf{T}\left(\beta x_{i}\right) \\
& -\frac{\omega^{2}}{6}\left(\frac{1}{3}\left(2+\cos \left(k_{i} \Delta_{x}\right)\right)+j \beta \frac{k_{i} \Delta_{x}^{2}}{6}\left(\cos \left(k_{i} \Delta_{x}\right) \frac{k^{\prime}\left(\beta x_{i}\right)}{k_{i}}+2 \sin _{c}\left(k_{i} \Delta_{x}\right) \frac{\left(d^{2}\left(\beta x_{i}\right) \mathbf{Y}\left(\beta x_{i}\right)\right)^{\prime}}{d_{i}^{2} \mathbf{Y}\left(\beta x_{i}\right)}\right)\right) d_{i}^{2} \mathbf{Y}\left(\beta x_{i}\right) \\
& +\frac{\left(\omega^{2}\right)}{2}\left(\frac{1}{3}\left(2+\cos \left(k_{i} \Delta_{x}\right)\right)+j \beta \frac{k_{i} \Delta_{x}^{2}}{6}\left(\cos \left(k_{i} \Delta_{x}\right) \frac{k^{\prime}\left(\beta x_{i}\right)}{k_{i}}+2 \sin _{c}\left(k_{i} \Delta_{x}\right) \frac{\left(d_{i}\left(\beta x_{i}\right) \mathbf{S}\left(\beta x_{i}\right)\right)^{\prime}}{d \mathbf{S}\left(\beta x_{i}\right)}\right) d_{i} \mathbf{S}\left(\beta x_{i}\right)=0\right.
\end{aligned}
$$

It remains to estimate the first order derivatives $\mathbf{U}^{\prime}\left(\beta x_{i}\right), \mathbf{T}^{\prime}\left(\beta x_{i}\right), \mathbf{Y}^{\prime}\left(\beta x_{i}\right)$ and $\mathbf{S}^{\prime}\left(\beta x_{i}\right)$.

First observe that $\mathbf{M} X=\mathbf{N} \bar{U}$ which provides, using (81)-(82),

$$
\begin{aligned}
& \left(\frac{1}{3}\left(2+\cos \left(k_{i} \Delta_{x}\right)\right)+j \beta \frac{k_{i} \Delta_{x}^{2}}{6}\left(\cos \left(k_{i} \Delta_{x}\right) \frac{k^{\prime}\left(\beta x_{i}\right)}{k_{i}}+2 \sin _{c}\left(k_{i} \Delta_{x}\right) \frac{\mathbf{X}^{\prime}\left(\beta x_{i}\right)}{\mathbf{X}\left(\beta x_{i}\right)}\right)\right) \mathbf{X}\left(\beta x_{i}\right) \\
& =\left(j k_{i} \sin _{c}\left(k_{i} \Delta_{x}\right)-\beta \frac{k_{i}^{2} \Delta_{x}^{2}}{2} \sin _{c}\left(k_{i} \Delta_{x}\right) \frac{k^{\prime}\left(\beta x_{i}\right)}{k_{i}}+\beta \cos \left(k_{i} \Delta_{x}\right) \frac{\mathbf{U}^{\prime}\left(\beta x_{i}\right)}{\mathbf{U}\left(\beta x_{i}\right)}\right) \mathbf{U}\left(\beta x_{i}\right)+\mathcal{O}\left(\beta^{2}\right) .
\end{aligned}
$$

Collecting the terms of order $\beta^{0}$ in the last expression, one gets

$$
\mathbf{X}\left(\beta x_{i}\right)=j k_{i} \frac{\sin _{c}\left(k_{i} \Delta_{x}\right)}{\frac{1}{3}\left(2+\cos \left(k_{i} \Delta_{x}\right)\right)} \mathbf{U}\left(\beta x_{i}\right)+\mathcal{O}(\beta) .
$$

Assuming that Equation (85) is valid for the continuous variable $x$, one derives by first applying the neperian logarithm and then by differentiating the resulting relation

$$
\frac{\mathbf{X}^{\prime}\left(\beta x_{i}\right)}{\mathbf{X}\left(\beta x_{i}\right)}=\frac{\mathbf{U}^{\prime}\left(\beta x_{i}\right)}{\mathbf{U}\left(\beta x_{i}\right)}+\left(\frac{\cos \left(k_{i} \Delta_{x}\right)}{\sin _{c}\left(k_{i} \Delta_{x}\right)}+\frac{k_{i}^{2} \Delta_{x}^{2}}{3} \frac{\sin _{c}\left(k_{i} \Delta_{x}\right)}{\frac{1}{3}\left(2+\cos \left(k_{i} \Delta_{x}\right)\right)}\right) \frac{k^{\prime}\left(\beta x_{i}\right)}{k_{i}}+\mathcal{O}(\beta)
$$


Plugging (86) in Equation (84), one obtains

$$
\begin{aligned}
& \mathbf{X}\left(\beta x_{i}\right)=j k_{i} \frac{\sin _{c}\left(k_{i} \Delta_{x}\right)}{\frac{1}{3}\left(2+\cos \left(k_{i} \Delta_{x}\right)\right)} \mathbf{U}\left(\beta x_{i}\right) \\
& +\beta\left[\left(\frac{\cos \left(k_{i} \Delta_{x}\right)}{\frac{1}{3}\left(2+\cos \left(k_{i} \Delta_{x}\right)\right)}+\frac{k_{i}^{2} \Delta_{x}^{2}}{3} \frac{\sin _{c}\left(k_{i} \Delta_{x}\right)^{2}}{\left[\frac{1}{3}\left(2+\cos \left(k_{i} \Delta_{x}\right)\right]^{2}\right.}\right) \frac{\mathbf{U}^{\prime}\left(\beta x_{i}\right)}{\mathbf{U}\left(\beta x_{i}\right)}\right. \\
& \left.+\left(\frac{k_{i}^{2} \Delta_{x}^{2}}{3} \frac{\sin _{c}\left(k_{i} \Delta_{x}\right)\left(\cos \left(k_{i} \Delta_{x}\right)-1\right)}{\left[\frac{1}{3}\left(2+\cos \left(k_{i} \Delta_{x}\right)\right)\right]^{2}}+\frac{k_{i}^{4} \Delta_{x}^{4}}{9} \frac{\sin _{c}\left(k_{i} \Delta_{x}\right)^{3}}{\left[\frac{1}{3}\left(2+\cos \left(k_{i} \Delta_{x}\right)\right)\right]^{3}}\right) \frac{k^{\prime}\left(\beta x_{i}\right)}{k_{i}}\right] \mathbf{U}\left(\beta x_{i}\right)+\mathcal{O}\left(\beta^{2}\right) .
\end{aligned}
$$

Following the same procedure, we express the variables $\mathbf{Z}$ and $\mathbf{W}$ in terms of $\mathbf{U}$ as follows

$$
\begin{aligned}
\mathbf{Z}\left(\beta x_{i}\right)= & j k_{i} d_{i} \frac{\sin _{c}\left(k_{i} \Delta_{x}\right)}{\frac{1}{3}\left(2+\cos \left(k_{i} \Delta_{x}\right)\right)} \mathbf{U}\left(\beta x_{i}\right)+\beta d_{i}\left[\beta d_{i}\left(\frac{k_{i}^{2} \Delta_{x}^{2}}{3} \frac{\sin _{c}\left(k_{i} \Delta_{x}\right)^{2}}{\left[\frac{1}{3}\left(2+\cos \left(k_{i} \Delta_{x}\right)\right]^{2}\right.}\right) \frac{d^{\prime}\left(\beta x_{i}\right)}{d_{i}}\right. \\
& +\left(\frac{\cos \left(k_{i} \Delta_{x}\right)}{\frac{1}{3}\left(2+\cos \left(k_{i} \Delta_{x}\right)\right)}+\frac{k_{i}^{2} \Delta_{x}^{2}}{3} \frac{\sin _{c}\left(k_{i} \Delta_{x}\right)^{2}}{\left[\frac{1}{3}\left(2+\cos \left(k_{i} \Delta_{x}\right)\right)\right]^{2}}\right) \frac{\mathbf{U}^{\prime}\left(\beta x_{i}\right)}{\mathbf{U}\left(\beta x_{i}\right)} \\
& \left.+\beta d_{i}\left(\frac{k_{i}^{2} \Delta_{x}^{2}}{3} \frac{\sin _{c}\left(k_{i} \Delta_{x}\right)\left(\cos \left(k_{i} \Delta_{x}\right)-1\right)}{\left[\frac{1}{3}\left(2+\cos \left(k_{i} \Delta_{x}\right)\right)\right]^{2}}+\frac{k_{i}^{4} \Delta_{x}^{4}}{9} \frac{\sin _{c}\left(k_{i} \Delta_{x}\right)^{3}}{\left[\frac{1}{3}\left(2+\cos \left(k_{i} \Delta_{x}\right)\right]^{3}\right.}\right) \frac{k^{\prime}\left(\beta x_{i}\right)}{k_{i}}\right] \mathbf{U}\left(\beta x_{i}\right) \\
& +\mathcal{O}\left(\beta^{2}\right),
\end{aligned}
$$

Recalling that $\mathcal{M} V=\mathcal{N}(D \diamond \bar{U})$, one can deduce the expression of $\mathbf{V}$ by substituting in (87) $\mathbf{X}$ by $\mathbf{V}$ and $\mathbf{U}$ by $\mathbf{d} \mathbf{U}$. Then $\mathbf{T}$ is computed directly by using the relation

$$
T=\left(D \diamond X+\frac{1}{3}(V-Z+2 W)\right) .
$$

Furthermore, $\mathbf{Y}$ and $\mathbf{S}$ are obtained using the relations $\mathcal{M} Y=\mathcal{N} X$ and $\mathcal{M} S=\mathcal{N} T$ and (87).

Coming back to (83), one obtains the expression of $\omega$, using the expressions of the amplitudes of $U, T, Y$ and $S$ and collecting in the resulting equation the term of order $\beta^{0}$, for all $i=0, \ldots, N$,

$$
\frac{\omega^{2}}{g d_{i} k_{i}^{2}}=\frac{\sin _{c}\left(k_{i} \Delta_{x}\right)^{2}}{\left[\frac{1}{3}\left(2+\cos \left(k_{i} \Delta_{x}\right)\right)\right]^{2}+\frac{k_{i}^{2} d_{i}^{2}}{3} \sin _{c}\left(k_{i} \Delta_{x}\right)^{2}} .
$$

Furthermore, collecting the term of order $\beta$, one obtains a relation between $\mathbf{U}, k$, and the bathymetry $d$ :

$$
\alpha_{1, i}^{(1)} \frac{\mathbf{U}^{\prime}\left(\beta x_{i}\right)}{\mathbf{U}_{i}}+\alpha_{2, i}^{(1)} \frac{d^{\prime}\left(\beta x_{i}\right)}{d_{i}}+\alpha_{3, i}^{(1)} \frac{k^{\prime}\left(\beta x_{i}\right)}{k_{i}}=0 .
$$

Equation (91) describes the effect of linear shoaling since the numbers $\alpha_{1, i}^{(1)}, \alpha_{2, i}^{(1)}$ and $\alpha_{3, i}^{(1)}$ are known as the linear shoaling coefficients. Using (90), one can compute these three coefficients (we omitt the details for simplicity), for all $i=0, \ldots, N$,

$$
\begin{gathered}
\alpha_{1, i}^{(1)}=2 \cos \left(k_{i} \Delta_{x}\right) \frac{\left[\frac{1}{3}\left(2+\cos \left(k_{i} \Delta_{x}\right)\right)\right]}{\sin _{c}\left(k_{i} \Delta_{x}\right)}+2 \frac{k_{i}^{2} \Delta_{x}^{2}}{3} \sin _{c}\left(k_{i} \Delta_{x}\right) \\
\alpha_{2, i}^{(1)}=2 \frac{1+2 \cos \left(k_{i} \Delta_{x}\right)}{3} \frac{\left[\frac{1}{3}\left(2+\cos \left(k_{i} \Delta_{x}\right)\right)\right]}{\sin _{c}\left(k_{i} \Delta_{x}\right)}-\frac{1+2 \cos \left(k_{i} \Delta_{x}\right)}{3} \frac{\sin _{c}\left(k_{i} \Delta_{x}\right)}{\left[\frac{1}{3}\left(2+\cos \left(k_{i} \Delta_{x}\right)\right)\right]} \frac{k_{i}^{2} d_{i}^{2}}{3}
\end{gathered}
$$

$\operatorname{RR} n^{\circ} 8806$ 


$$
\begin{aligned}
& \alpha_{3, i}^{(1)}=\frac{\cos \left(k_{i} \Delta_{x}\right)^{2}}{\sin _{c}\left(k_{i} \Delta_{x}\right)^{2}}\left[\frac{1}{3}\left(2+\cos \left(k_{i} \Delta_{x}\right)\right)\right]+2 \frac{k_{i}^{2} \Delta_{x}^{2}}{3}\left(\cos \left(k_{i} \Delta_{x}\right)-1\right)+\frac{k_{i}^{4} \Delta_{x}^{4}}{9} \frac{\sin _{c}\left(k_{i} \Delta_{x}\right)^{2}}{\left[\frac{1}{3}\left(2+\cos \left(k_{i} \Delta_{x}\right)\right)\right]} \\
& +\frac{k_{i}^{2} d_{i}^{2}}{3}\left[\frac{\cos \left(k_{i} \Delta_{x}\right)^{2}}{2 \sin _{c}\left(k_{i} \Delta_{x}\right)}\left(\frac{\left[\frac{1}{3}\left(2+\cos \left(k_{i} \Delta_{x}\right)\right)\right]^{2}}{\sin _{c}\left(k_{i} \Delta_{x}\right)}-1\right)+2 \frac{k_{i}^{4} \Delta_{x}^{4}}{9} \frac{\sin _{c}\left(k_{i} \Delta_{x}\right)^{2}\left(1-\cos \left(k_{i} \Delta_{x}\right)\right)}{\left[\frac{1}{3}\left(2+\cos \left(k_{i} \Delta_{x}\right)\right)\right]^{2}}\right] .
\end{aligned}
$$

Differentiating formally the dispersion relation (90) and assuming that $\omega$ is constant, we deduce that $k_{i}$ has to satisfy the following condition

$$
\frac{k^{\prime}\left(\beta x_{i}\right)}{k_{i}}=-\alpha_{4}^{(1)} \frac{d^{\prime}\left(\beta x_{i}\right)}{d_{i}}
$$

where, for all $i=0, \ldots, N$,

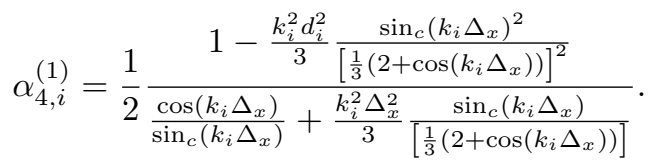

This relation is used to compute formally $k_{i}$ for $i=0, \ldots, N$ and for a given bathymetry and therefore to obtain the coefficients $\alpha_{1, i}^{(1)}, \alpha_{2, i}^{(1)}$ and $\alpha_{3, i}^{(1)}$. Finally, following [6], we obtain an expression of the amplitude of the surface elevation $A$, for all $i=0, \ldots, N$

where

$$
\frac{A_{i}^{\prime}}{A_{i}}=-\alpha_{s, i}^{(1)} \frac{d_{i}^{\prime}}{d_{i}}
$$

$$
\alpha_{s, i}^{(1)}=\frac{\alpha_{2, i}^{(1)}-\left(\alpha_{3, i}^{(1)}+\alpha_{1, i}^{(1)}\right) \alpha_{4, i}^{(1)}}{\alpha_{1, i}^{(1)}} .
$$

\subsection{Linear characteristics of the classical Peregrine model.}

We consider now the classical numerical model presented in Section 3. We reproduce the same procedure as in Section 5.1. The terms of order of $\beta^{0}$ gives the linear dispersion relation, for all $i=0 \ldots N$,

$$
\frac{\omega^{2}}{g d_{i} k_{i}^{2}}=\frac{\sin _{c}\left(k_{i} \Delta_{x}\right)^{2}}{\frac{1}{3}\left(2+\cos \left(k_{i} \Delta_{x}\right)\right)\left(\frac{1}{3}\left(2+\cos \left(k_{i} \Delta_{x}\right)\right)+\frac{1-\cos \left(k_{i} \Delta_{x}\right)}{\frac{k_{i}^{2} \Delta_{x}^{2}}{2}} \frac{k_{i}^{2} d_{i}^{2}}{3}\right)},
$$

whereas the terms of order of $\beta$ provides the linear shoaling coefficients as follows

$$
\begin{aligned}
& \alpha_{1, i}^{(2)}=2\left[\frac{1}{3}\left(2+\cos \left(k_{i} \Delta_{x}\right)\right)\right] \sin _{c}\left(k_{i} \Delta_{x}\right) \cos \left(k_{i} \Delta_{x}\right)+ \\
& 2 \frac{k_{i}^{2} d_{i}^{2}}{3}\left(\sin _{c}\left(k_{i} \Delta_{x}\right) \cos \left(k_{i} \Delta_{x}\right) \frac{1-\cos \left(k_{i} \Delta_{x}\right)}{\frac{k_{i}^{2} \Delta_{x}^{2}}{2}}-\sin _{c}\left(k_{i} \Delta_{x}\right)^{3}\right) \\
& +\frac{k_{i}^{2} \Delta_{x}^{2}}{3}\left(2 \sin _{c}\left(k_{i} \Delta_{x}\right)^{3}+\frac{k_{i}^{2} d_{i}^{2}}{3} \frac{\sin _{c}\left(k_{i} \Delta_{x}\right)^{3}}{\frac{1}{3}\left(2+\cos \left(k_{i} \Delta_{x}\right)\right)} \frac{1-\cos \left(k_{i} \Delta_{x}\right)}{\frac{k_{i}^{2} \Delta_{x}^{2}}{2}}\right) \\
& \alpha_{2, i}^{(2)}=\left[\frac{1}{3}\left(2+\cos \left(k_{i} \Delta_{x}\right)\right)\right] \sin _{c}\left(k_{i} \Delta_{x}\right) \frac{5 \cos \left(k_{i} \Delta_{x}\right)+1}{3} \\
& +\frac{k_{i}^{2} d_{i}^{2}}{3}\left(\frac{1-\cos \left(k_{i} \Delta_{x}\right)}{\frac{k_{i}^{2} \Delta_{x}^{2}}{2}} \sin _{c}\left(k_{i} \Delta_{x}\right) \frac{5 \cos \left(k_{i} \Delta_{x}\right)+1}{3}-3 \sin _{c}\left(k_{i} \Delta_{x}\right)\right) \\
& +\frac{k_{i}^{2} \Delta_{x}^{2}}{3}\left(\sin _{c}\left(k_{i} \Delta_{x}\right)^{3}+\frac{k_{i}^{2} d_{i}^{2}}{3} \frac{\sin _{c}\left(k_{i} \Delta_{x}\right)^{3}}{\left[\frac{1}{3}\left(2+\cos \left(k_{i} \Delta_{x}\right)\right)\right]} \frac{1-\cos \left(k_{i} \Delta_{x}\right)}{\frac{k_{i}^{2} \Delta_{x}^{2}}{2}}\right)
\end{aligned}
$$




$$
\begin{aligned}
& \alpha_{3, i}^{(2)}=\left[\frac{1}{3}\left(2+\cos \left(k_{i} \Delta_{x}\right)\right)\right] \cos \left(k_{i} \Delta_{x}\right)^{2}+\frac{k_{i}^{2} d_{i}^{2}}{3}\left(\frac{1-\cos \left(k_{i} \Delta_{x}\right)}{\frac{k_{i}^{2} \Delta_{x}^{2}}{2}} \cos \left(k_{i} \Delta_{x}\right)^{2}\right. \\
& \left.-\sin _{c}\left(k_{i} \Delta_{x}\right)^{2} \cos \left(k_{i} \Delta_{x}\right)\right)+\frac{k_{i}^{2} \Delta_{x}^{2}}{6}\left(4 \sin _{c}\left(k_{i} \Delta_{x}\right)^{2} \cos \left(k_{i} \Delta_{x}\right)-3 \sin _{c}\left(k_{i} \Delta_{x}\right)^{2}\right. \\
& \left.-k_{i}^{2} d_{i}^{2} \frac{1-\cos \left(k_{i} \Delta_{x}\right)}{\frac{k_{i}^{2} \Delta_{x}^{2}}{2}} \frac{\sin _{c}\left(k_{i} \Delta_{x}\right)^{2}}{\frac{1}{3}\left(2+\cos \left(k_{i} \Delta_{x}\right)\right)}\right)+\frac{k_{i}^{4} d_{i}^{4}}{9}\left(\frac{\sin _{c}\left(k_{i} \Delta_{x}\right)^{4}}{\frac{1}{3}\left(2+\cos \left(k_{i} \Delta_{x}\right)\right)}\right. \\
& \left.+\frac{k_{i}^{2} d_{i}^{2}}{3} \frac{\sin _{c}\left(k_{i} \Delta_{x}\right)^{4}}{\left[\frac{1}{3}\left(2+\cos \left(k_{i} \Delta_{x}\right)\right]^{2}\right.} \frac{1-\cos \left(k_{i} \Delta_{x}\right)}{\frac{k_{i}^{2} \Delta_{x}^{2}}{2}}\right) .
\end{aligned}
$$

Differentiating Equation (95), assuming that $\omega$ is constant, we obtain the relation that must satisfies each $k_{i}$ for all $i=0, \ldots, N$

$$
\frac{k_{i}^{\prime}}{k_{i}}=-\alpha_{4, i}^{(2)} \frac{d_{i}^{\prime}}{d_{i}}
$$

where

$$
\alpha_{4, i}^{(2)}=\frac{1}{2 C}\left(\frac{\frac{1}{3}\left(2+\cos \left(k_{i} \Delta_{x}\right)\right)}{\cos \left(k_{i} \Delta_{x}\right)}-\frac{k_{i}^{2} d_{i}^{2}}{3} \frac{1-\cos \left(k_{i} \Delta_{x}\right)}{\cos \left(k_{i} \Delta_{x}\right) \frac{k_{i}^{2} \Delta_{x}^{2}}{2}}\right)
$$

and

$$
\begin{gathered}
C=\frac{\frac{1}{3}\left(2+\cos \left(k_{i} \Delta_{x}\right)\right)}{\sin _{c}\left(k_{i} \Delta_{x}\right)}+\frac{k_{i}^{2} d_{i}^{2}}{3}\left(\frac{1-\cos \left(k_{i} \Delta_{x}\right)}{\sin _{c}\left(k_{i} \Delta_{x}\right) \frac{k_{i}^{2} \Delta_{x}^{2}}{2}}-\frac{\sin _{c}\left(k_{i} \Delta_{x}\right)}{\cos \left(k_{i} \Delta_{x}\right)}\right) \\
+\frac{k_{i}^{2} \Delta_{x}^{2}}{3 \cos \left(k_{i} \Delta_{x}\right)}\left[\sin _{c}\left(k_{i} \Delta_{x}\right)+\frac{k_{i}^{2} d_{i}^{2}}{6} \frac{\sin _{c}\left(k_{i} \Delta_{x}\right)\left(1-\cos \left(k_{i} \Delta_{x}\right)\right)}{\frac{1}{3}\left(2+\cos \left(k_{i} \Delta_{x}\right)\right) \frac{k_{i}^{2} \Delta_{x}^{2}}{2}}\right] .
\end{gathered}
$$

Again, we obtain the relation between the amplitude $A$ of the surface elevation and the bathymetry which reads, for all $i=1, \ldots, N$,

$$
\frac{A_{i}^{\prime}}{A_{i}}=-\alpha_{s, i}^{(2)} \frac{d_{i}^{\prime}}{d_{i}}
$$

where

$$
\alpha_{s, i}^{(2)}=\frac{\alpha_{2, i}^{(2)}-\left(\alpha_{3, i}^{(2)}+\alpha_{1, i}^{(2)}\right) \alpha_{4, i}^{(2)}}{\alpha_{1, i}^{(2)}} .
$$

\subsection{Analysis of the computations.}

In this section, we study the linear dispersion relations derived in Section 5.1 and 5.2. More precisely, we draw the phase velocity and the amplitude of the wave with respect to the dispersion parameter $\sigma$ in shoaling conditions for each scheme and we compare the results with the ones predicted by the linear theory associated to the Peregrine equations (1).

\subsubsection{Phase velocity}

For a given numerical scheme, the phase velocity is usually given by the relation

$$
C=\frac{\omega}{k}
$$

$\mathrm{RR} \mathrm{n}^{\circ} 8806$ 
As observe in literature, we can consider $k$ and $d$ as constant functions $\left(k=k_{0}\right.$, and $d=d_{0}$ see [17] for more details). Then, the phase velocity of our new numerical scheme (75)-(76) is

$$
C_{(1)}^{2}=g d \frac{\sin _{c}\left(k \Delta_{x}\right)^{2}}{\left[\frac{1}{3}\left(2+\cos \left(k \Delta_{x}\right)\right)\right]^{2}+\frac{k^{2} d^{2}}{3} \sin _{c}\left(k \Delta_{x}\right)^{2}},
$$

while that derived for the classical scheme (11)-(12) reads

$$
C_{(2)}^{2}=g d \frac{\sin _{c}\left(k \Delta_{x}\right)^{2}}{\frac{1}{3}\left(2+\cos \left(k \Delta_{x}\right)\right)\left(\frac{1}{3}\left(2+\cos \left(k \Delta_{x}\right)\right)+\frac{1-\cos \left(k \Delta_{x}\right)}{\frac{k^{2} \Delta_{x}^{2}}{2}} \frac{k^{2} d^{2}}{3}\right)} .
$$

Our aim is to plot the two curves (98)-(99) and compare with the one predicted by the linear theory :

$$
C_{P}^{2}=\sqrt{\frac{g d}{1+\frac{k^{2} d^{2}}{3}}} .
$$

We first fix the wavelength $\lambda$ and we put $\Delta_{x}=\frac{\lambda}{N_{\lambda}}$ where $N_{\lambda}$ is the number of discretization points by wavelength. A direct computation gives :

$$
k \Delta_{x}=\frac{2 \pi}{N_{\lambda}} .
$$

We recall that $\sigma=\frac{d}{\lambda}$, showing that $k d=2 \pi \sigma$. In Figure 2, we draw the relative errors between (98) and (99) and the phase velocity predicted by the linear theory. The error er is defined, for each scheme, by

$$
\text { er }=100\left(\frac{C-C_{P}}{C_{P}}\right)
$$
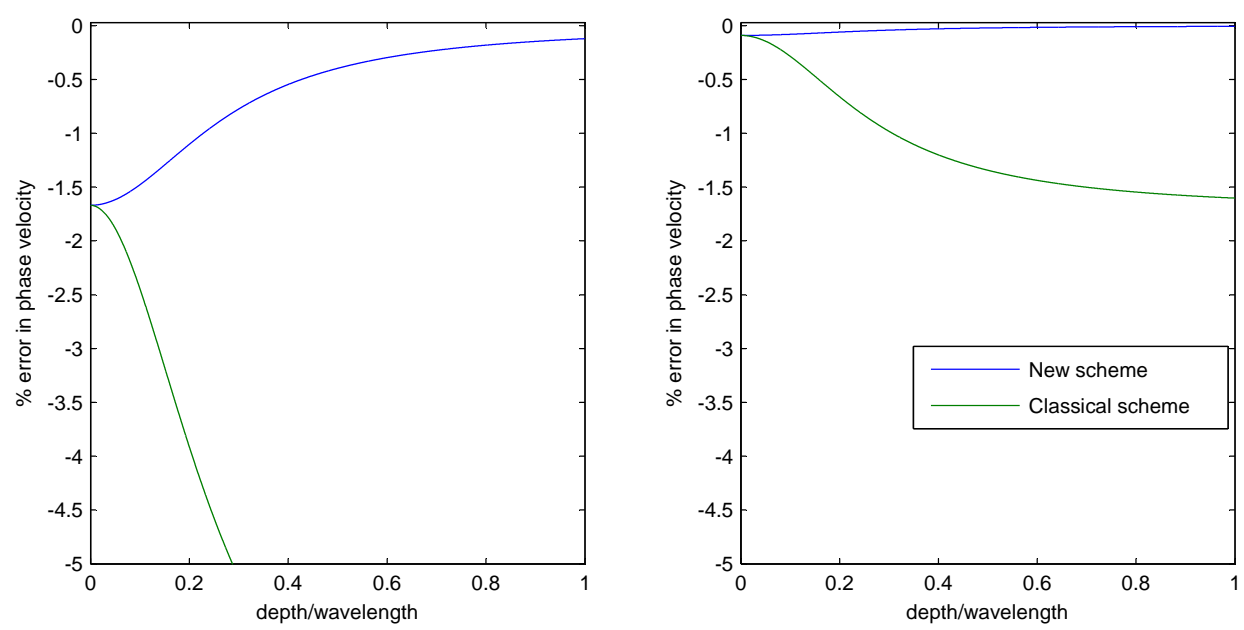

Figure 2: Comparison of the phase velocity $\left(N_{\lambda}=5\right.$ on the left, $N_{\lambda}=10$ on the right) of the classical and the new numerical scheme with the one given by the linear theory w.r.t $\sigma$.

In Figure 2, one can observe that for $N_{\lambda}=5$, the error coming from the new scheme is acceptable (less than 1.6\%) whereas the one of the classical scheme is greater than $5 \%$ for 
depths bigger than 0,3 . For $N_{\lambda}=10$, although the error of the classical scheme is better than in the previous case (less than $2 \%$ ), the one of the new scheme is much better and stay very close to 0 . We conclude here that our new numerical scheme seems to reproduce much better the linear dispersive effects.

\subsubsection{Linear shoaling test}

We first recall the expression of the shoaling coefficients given by the linear theory associated with the Peregrine equations (1) (see [6]):

$$
\alpha_{1}=2, \alpha_{2}=2-\frac{k^{2} d^{2}}{3}, \alpha_{3}=1, \alpha_{4}=\frac{1}{2}\left(1-\frac{k^{2} d^{2}}{3}\right)
$$

Our aim is to compare, for a given situation, the evolution of the amplitude of the waves with respect to the space variable $x$ given by the two relations (93) and (97) and the one derived from the linear theory. We consider a periodic wave with an initial amplitude $a=0.05$ and a wavelength $\lambda=15$ starting from the position $x=100$. It propagates over an initial constant water depth $d_{0}=13$. The bottom is flat until $x=150$ and it has a constant up-slope of $\frac{1}{50}$ from $x=150$ to $x=790$. We compute the evolution of the wave amplitude with respect to $x$. For that, we propose the following procedure. Firstly, we integrate formally the relation between $k$ and $d((92)$ for the new scheme and (96) for the classical one), given by the differential equation

$$
\frac{k^{\prime}}{k}=-\alpha_{4} \frac{d^{\prime}}{d}
$$

We use a Strongly Stability-Preserving Runge-Kutta method (SSP-RK) to compute the solution $k$, using $k_{0}=\frac{2 \pi}{\lambda}$ as initial condition. Then, we substitute this function $k$ in the expression of $\alpha_{s}$, and we compute the amplitude of the wave $A$ by integrating the relation ((93) for the new scheme and (97) for the classical one)

$$
\frac{A^{\prime}}{A}=-\alpha_{s} \frac{d^{\prime}}{d}
$$

Again, we use a SSP-RK method and $A(100)=0.05$ as initial condition. Fixing the value of $\Delta_{x}$, it is possible to compute formally the surface elevation amplitude for each scheme. The results are presented in Figure 3. 

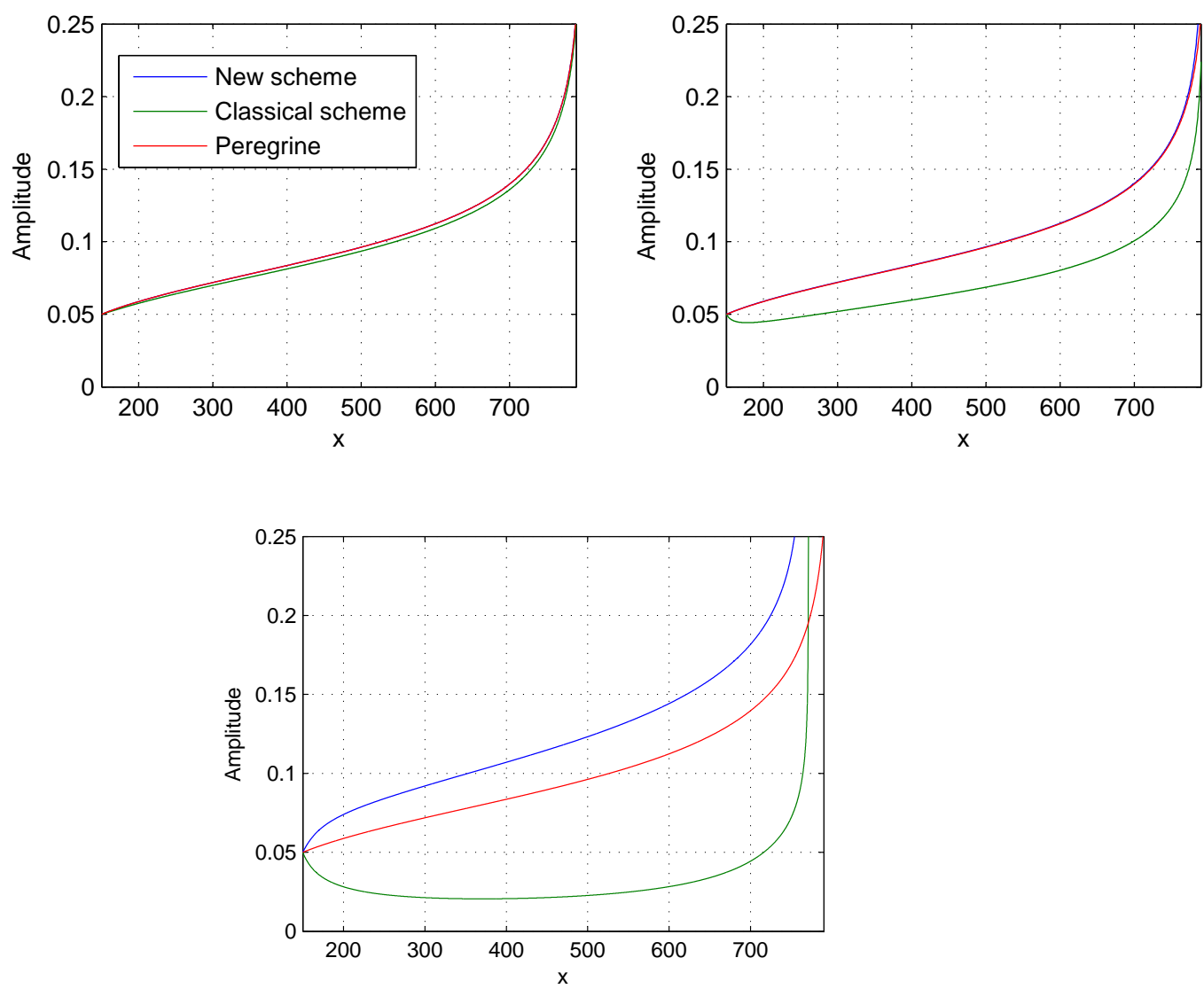

Figure 3: Evolution of the wave amplitude for the two numerical schemes and for Peregrine equations. Top left : $\Delta_{x}=0,5\left(N_{\lambda}=30\right)$. Top right : $\Delta_{x}=1,5\left(N_{\lambda}=10\right)$. Down : $\Delta_{x}=3\left(N_{\lambda}=5\right)$

We observe that when $\Delta_{x}$ is small, the curves of the two schemes matched the theoretical one, meaning that both schemes converges. However, when $\Delta_{x}$ becomes larger, one can see that the curve computed with the new scheme stay closed to the theoretical one while the one computed with the classical scheme underestimates drastically the amplitude of the wave.

\section{Numerical experiments}

This section is devoted to the investigation of the nonlinear behavioir of the two schemes (73)-(74) and (11)-(12). To this end, we present two different test cases : the propagation of solitary waves over a flat bathymetry and a shoaling test for which some solitary wave profile is computed in a situation close to wave breaking (see [10] for details). These test cases bring to the fore major differences between the two numerical models and confirm the premiminary results of Section 5 . 


\subsection{Soliton propagation}

We first consider the propagation of an exact solitary wave solution to the Peregrine equations, with an amplitude equal to 0,2 (details on the computation of this solution as well as mathematical conditions for the existence are given in [3]) over a flat bathymetry $d_{0}=1$. The space intervall is equal to $[0,100]$. In order to check our implementations, we have performed a grid convergence analysis. Numerical results have been compared with the initial profile (which is the profile of the exact solution). The meshes used contain respectively 1000, 2000, 4000 and 8000 points. In Figure 4, we have plotted the $L^{2}$-norm of the rescaled error for each scheme. The slope obtained for the scheme (73)-(74) shows a convergence of order 2 while that corresponding to (11)-(12) is equal to 1.7. We deduce that with the same initial finite elements method, the new procedure improves the order of convergence.

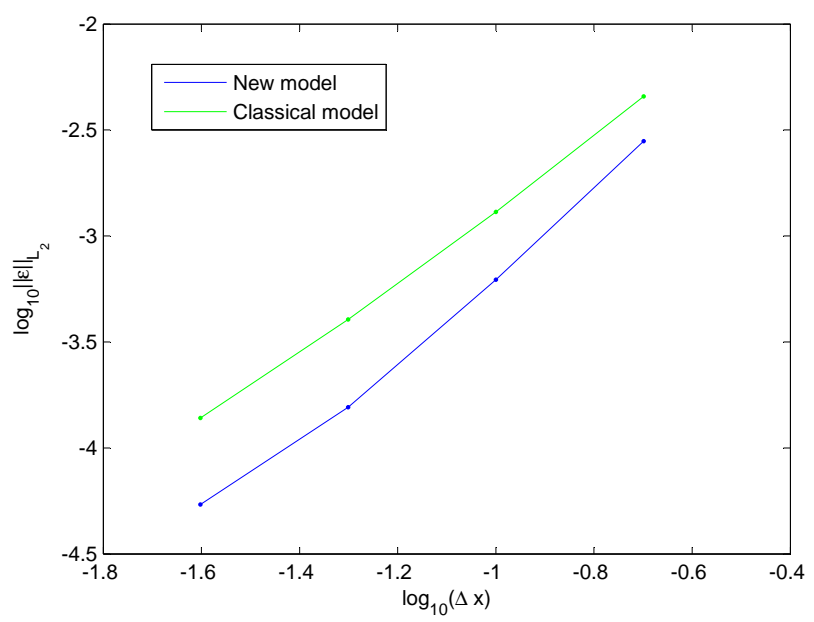

Figure 4: Grid convergence results for the two numerical schemes.

\subsection{Shoaling test}

This test case is dedicated to the wave shoaling characteristics of the two models considered here in conditions close to wave breaking. It has been initially proposed by Grilli et al. in [10] and consists in propagating a solitary wave of amplitude $0.2 \mathrm{~m}$ on a water depth $d_{0}=1 \mathrm{~m}$ during a time $13 \mathrm{~s}$. The shoaling takes place onto a constant slope of $\frac{1}{35}$ (see Figure 5).

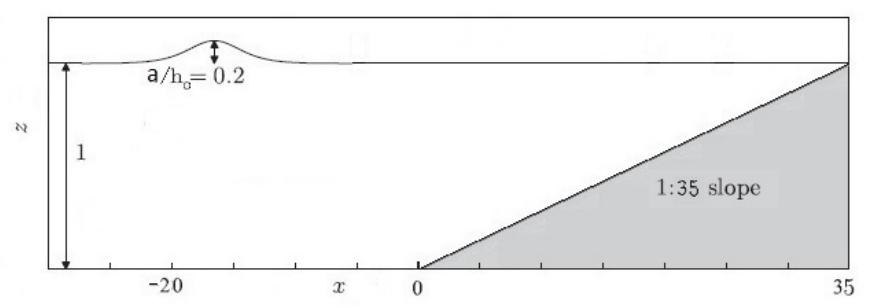

Figure 5: Shoaling of a solitary wave; computational configuration (Grilli and $a l$ ). 
Note that, to our knowledge, it is not possible to give an analytical solution in this configuration. We then decide to compute a reference solution using a very refined mesh of 8000 points and the scheme (11)-(12). This solution is used as a standard in the sequel to make the comparisons.

In Figure 6, we have plotted the shoaling wave profile using succesively a mesh containing 250 points (left side) and 500 points (right side) for the two numerical schemes as well as the reference solution.
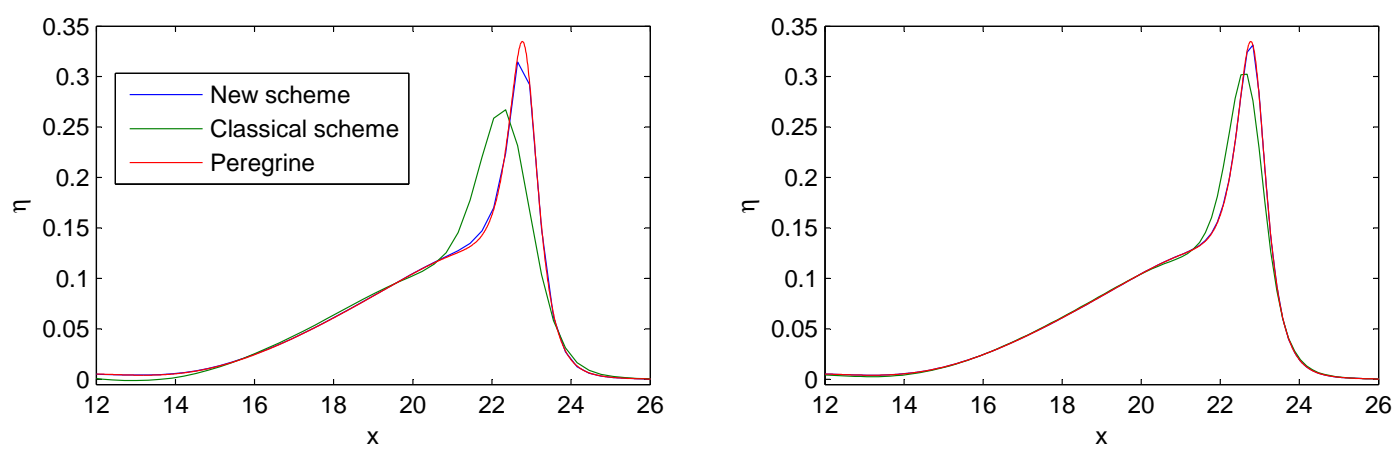

Figure 6: Shoaling wave profiles of Peregrine schemes. Left : mesh with 250 points. Right : mesh with 500 points

Clearly, one can observe a major difference in the behaviour of the two schemes. For a mesh with 250 points $\left(\Delta_{x}=0,3\right)$, the solution emanating from the new scheme (73)-(74) matches very well the reference curve, showing that the convergence as already occurs with a few numbers of points while it is obviously not the case for the scheme (11)(12). Indeed, the green curve exhibit some amplitude and phase defects. To push on the analysis, we repeat the same computations with a grid mesh containing 500 points $\left(\Delta_{x}=0,15\right)$. Again, we can see that the solution computed with the scheme (73)-(74) coincides perfectly with the reference curve. However, in this case, one can observe that the classical scheme provides better results without reaching the precision of the other scheme. Note that the conclusion doesn't changed if one computes the reference solution with the scheme (73)-(74). In addition, the Table 7 contains the relative errors in $L^{2}$ and $L^{\infty}$ norms for each scheme, where the errors are defined by

$$
\mathcal{E}_{2}=\frac{\left\|E-E_{\text {ref }}\right\|_{2}}{\left\|E_{\text {ref }}\right\|_{2}}, \mathcal{E}_{\infty}=\left|\frac{\|E\|_{\infty}-\left\|E_{\text {ref }}\right\|_{\infty}}{\left\|E_{r e f}\right\|_{\infty}}\right|
$$

$E_{\text {ref }}$ being the reference solution and $E$ the one of the two numerical schemes. The computations have been made with respectively 250, 500, 1000 and 2000 points. 


\begin{tabular}{|l|cccc|}
\hline Relative error $\backslash$ Points per meshes & 250 & 500 & 1000 & 2000 \\
\hline $\mathcal{E}_{2}$ for the New scheme & $1.55 \%$ & $0.34 \%$ & $0.09 \%$ & $0.03 \%$ \\
$\mathcal{E}_{2}$ for the Classical scheme & $31.25 \%$ & $12.67 \%$ & $3.70 \%$ & $0.81 \%$ \\
$\mathcal{E}_{\infty}$ for the New scheme & $6.01 \%$, & $1.03 \%$ & $0.07 \%$ & $0.04 \%$ \\
$\mathcal{E}_{\infty}$ for the Classical scheme & $20.19 \%$ & $9.67 \%$ & $3.31 \%$ & $0.85 \%$ \\
\hline
\end{tabular}

Figure 7: Relatives errors between the two numerical schemes and the reference solution for meshes with 250, 500,1000 and 2000 points

It transpires that the new scheme gives results very close to the exact solution, whereas the classical one need more points to give similar approximations. Finally, to compare the accuracy of the two models, we have performed a grid convergence analysis. In Figure 8, we have plotted the error defined in (101) in the $L^{2}$-norm for each scheme, corresponding to succesively 500, 1000 and 2000 points.

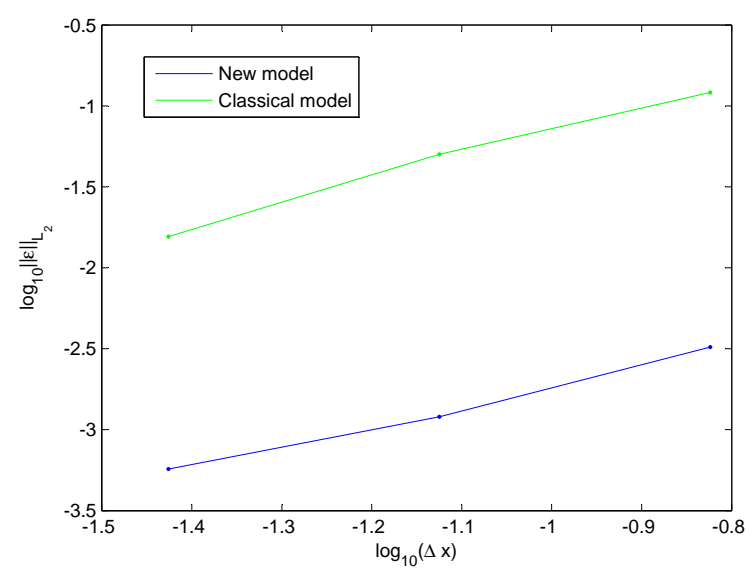

Figure 8: Grid convergence results for the shoaling of the two numerical schemes.

The slopes obtained for the error are similar for the two schemes. However, it is clear that the new model gives better results with 500 points that the classical scheme with 2000 points. We can deduce from this analysis that the new numerical scheme better reproduce physical effects and dispersion characteristics.

\section{Conclusions and perspectives}

We have presented a new systematic method to obtain discrete numerical model in the study of incompressible free surface flows. In order to evaluate the power of this method, we have considered the case of the so-called Peregrine equations and performed the computations in this academic situation. We have compared our new numerical scheme with the one obtained by performing directly a Galerkin method on the Peregrine equations. 
Finally, by the use of several numerical experiments, we have shown the efficiency of our new scheme.

Moreover, we claim that it is possible to obtain other different numerical scheme, consistent with the Peregrine equations, by changing the initial discretization of the Euler equations. For example, by choosing the following discrete boundary conditions :

- At the free surface :

$$
\hat{W}=E_{t}+\mathcal{K}(E \diamond \hat{U})-E \diamond(\mathcal{K} \hat{U}) .
$$

- On the seafloor :

$$
\check{W}=-(\mathcal{K}(D \diamond \check{U})-D \diamond(\mathcal{K} \check{U})),
$$

we are able to derive a new numerical scheme in a conservative form

$$
\begin{gathered}
E_{t}+\mathcal{K}(H \diamond \bar{U})=0, \\
\bar{U}_{t}+\left(\mathcal{K} \bar{U}^{2}-\bar{U} \diamond(\mathcal{K} \bar{U})\right)+g \mathcal{K} E+\left[\frac{D^{2}}{6} \diamond\left(\mathcal{K}^{2} \bar{U}_{t}\right)-\frac{D}{2} \diamond\left(\mathcal{K}^{2}\left(D \diamond \bar{U}_{t}\right)\right)\right]=0 .
\end{gathered}
$$

In the future, we plan to apply this new procedure to derive numerical schemes for Extended Boussinesq's models as well as for the Green-Naghdi equations. By this procedure, one of our goal is, for example, to enhance the linear dispersion characteristics of these models.

\section{Acknowledgment}

This study has been carried out with financial support from the French State, managed by the French National Research Agency (ANR) in the frame of the "Investments for the future" Programme IdEx Bordeaux - CPU (ANR-10-IDEX-03-02). Partial funding by the TANDEM contract (reference ANR-11-RSNR-0023-01 French Programme Investissements d'Avenir) is also acknowledged.

\section{References}

[1] R Abgrall and R Saurel, Discrete equations for physical and numerical compressible multiphase mixtures, Journal of Computational Physics, 186 (2003), pp. 361-396.

[2] S. Beji and K. Nadaoka, A formal derivation and numerical modeling of the improved Boussinesq equations for varying depth, Ocean Engineering, 23 (1996), pp. 691-704.

[3] S. Bellec and M. Colin : On the existence of solitary waves for Boussinesq type equations and a new conservative model. Preprint.

[4] P. Bonneton and F. Chazel and D. Lannes and F. Marche and M. Tissier: A splitting approach for the fully nonlinear and wakly dispersive Green-Naghdi model. Journal of Computational Physics, 230 (2011), pp. 1479-1498.

[5] M. Brocchini: A reasoned overview on Boussinesq-type models: the interplay between physics, mathematics and numerics. Proc. R. Soc. A, 469 (2013), 20130496.

[6] M.W. Dingemans: Water wave propagation over uneven bottoms. Advanced Series Ocean Eng., (1997), World Scientific. 
[7] A. Ern and J.-C. Guermond: Theory and practice of finite elements, Applied Mathematical Sciences Vol. 159, Springer (2004)

[8] C. Eskilsson and S.J. Sherwin: Spectral/HP discontinuous Galerkin methods for modelling 2D Boussinsq equations Journal of Computational Physics, 212 (2006), pp. 566-589.

[9] A. Filippini, S. Bellec, M. Colin and M. Ricchiuto : On the nonlinear behaviour of Boussinesq type models : amplitude-velocity vs amplitude-flux forms. Coastal Engineering, 99 (2014), pp. 109-123.

[10] S.T. Grilli, R. Subramanya, I.A. Svendsen and J. Veeramony: Shoaling of solitary waves on plane beaches. J.Waterw.Port.C.-ASCE, 120 (1994), pp. 609-628.

[11] M. Kazolea and A.I. Delis and I.K. Nikolos and C.E. Synolakis: An unstructured finite volume numerical scheme for extended 2D Boussinesq-type equations. Coastal Engineering, 69 (2012), pp. 42-66.

[12] D. Lannes and P. Bonneton, Derivation of asymptotic two-dimensional timedependent equations for surface water wave propagation, Physics of fluids, 21 (2009), 016601.

[13] A. Madsen, R.Murray and O.R. Sorensen, A new form of the Boussinesq equations with improved linear dispersion characteristics, Coastal engineering, 15 (1991), pp. 371-388.

[14] O. Nwogu, Alternative form of Boussinesq equations for nearshore wave propagation, ASCE Journal of Waterway, Port, Coastal and Ocean Engineering, (1993), 618-638.

[15] D.H. Peregrine, Long waves on a beach. J.Fluid.Mech, 27 (1967), pp. 815-827.

[16] M. Ricchiuto and A.G. Filippini: Residual discretization of enhanced Boussinesq equations for wave propagation over complex bathymetries. J. Comput. Phys., 271 (2014), pp. 306-341.

[17] M.K. Walkley, A Numerical Method for Extended Boussinesq Shallow-Water Wave Equations, PhD University of Leeds, (1999).

[18] M.A. Walkley And M. Berzins, A finite element method for the two-dimensional extended Boussinesq equations, Int. J. Numer. Meth Fluids, 39 (2002), pp. 865-885. 


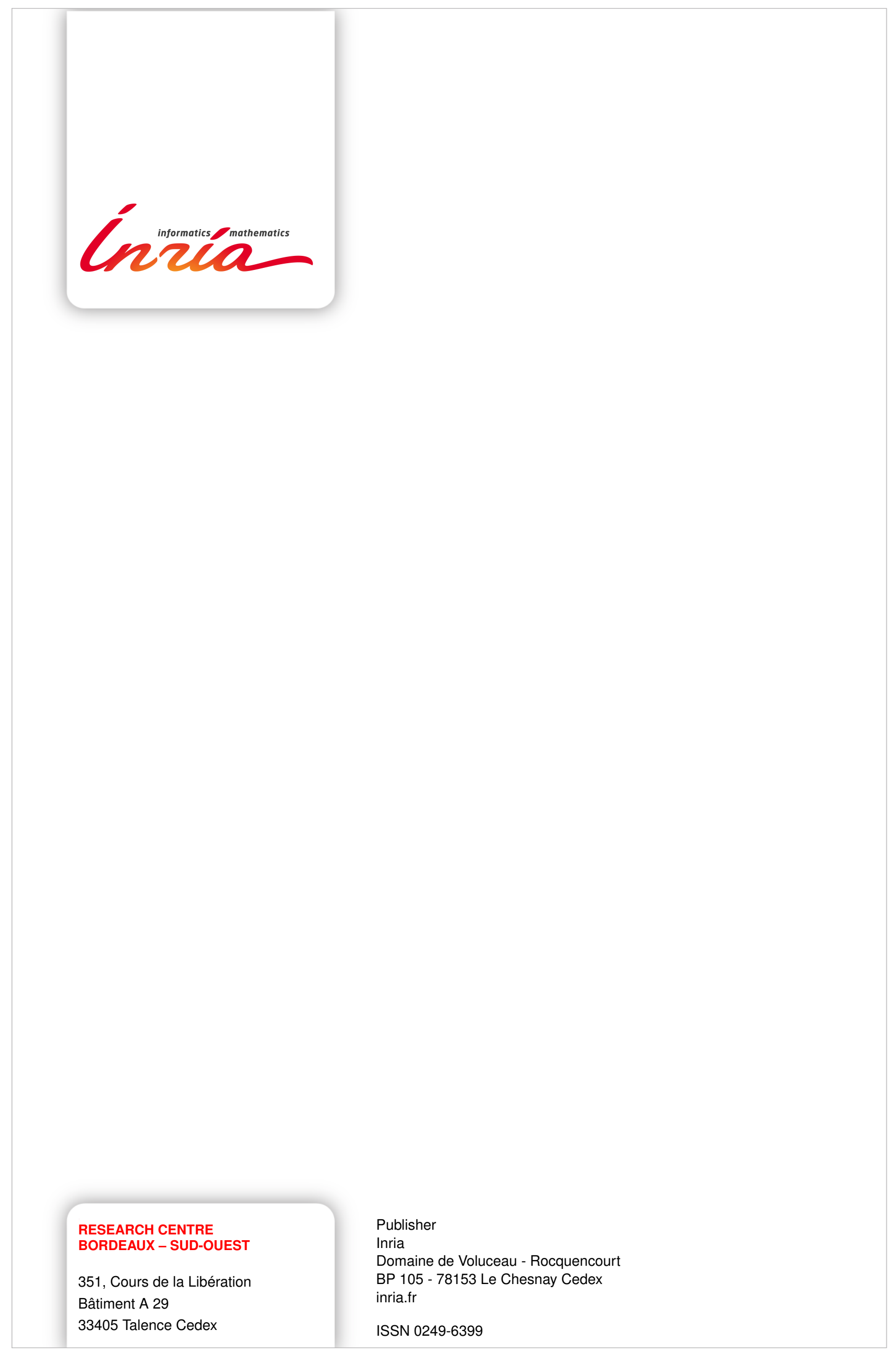

